Chinese Journal of Organic Chemistry

\title{
炔酰胺合成方法研究进展
}

\author{
赵永丽 $a$ 涂永良 ${ }^{a}$ 蔡明中 $a$ 赵军锋 $* b$ \\ ( ${ }^{a}$ 江西师范大学化学化工学院 南昌 330022) \\ (b 广州医科大学药学院 广州 511436)
}

\begin{abstract}
摘要 炔酰胺是一类氮原子上连有吸电子基团的特殊炔胺. 吸电子基团的引入使炔酰胺的稳定性和反应活性达到较好 的平衡, 从而赋予炔酰胺丰富多样的反应性能. 近二十年炔酰胺化学发展迅速, 其合成方法已引起广泛关注. 在此背 景下, 综述了炔酰胺合成方法的研究进展. 根据使用主要原料的结构, 分别从以卤代烯酰胺、炔烃以及其衍生物和卤代 烯烃为关键原料的合成方法进行了阐述，并对代表性反应机理进行了讨论.
\end{abstract}

关键词 炔酰胺; 吸电子基团; 内炔酰胺; 端炔酰胺

\section{Recent Advances in the Synthesis of Ynamides}

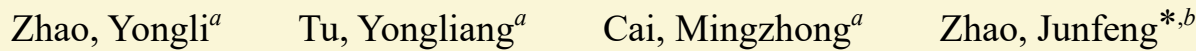 \\ ( ${ }^{a}$ College of Chemistry and Chemical Engineering, Jiangxi Normal University, Nanchang 330022) \\ ( ${ }^{b}$ School of Pharmaceutical Sciences, Guangzhou Medical University, Guangzhou 511436)
}

\begin{abstract}
Ynamides are a class of special ynamines bearing an electron-withdrawing group on the nitrogen atom. The electron-withdrawing group endows ynamides an optimal balance between the stability and activity, and thus enabling ynamides to be versatile synthons widely used in organic synthetic chemistry. The ynamide chemistry has witnessed a rapid development over the past two decades. As a consequence, its synthetic methods have attracted widespread attention. In this context, the recent advances in the synthesis of ynamides are reviewed herein. According to the structure of the main starting materials, the contents are classified as the synthesis with haloenamides, alkynes or its derivatives, and dihalo-substituted alkenes. The representative mechanisms are also discussed.

Keywords ynamide; electron-withdrawing group; internal ynamide; terminal ynamide
\end{abstract}

炔胺是一种特殊的含氮原子的炔烃，其分子结构中 氮原子与三键直接相连.独特的结构使炔胺在反应中具 有很高的反应活性和良好的区域选择性，因此经常用来 解决天然产物和有机合成中区域选择性和立体选择性 问题 ${ }^{[1]}$.

1958 年, Zaugg 等 ${ }^{2]}$ 首次报道了炔胺的合成(Scheme 1), 但过高的反应活性阻碍了炔胺的进一步发展. 后续 化学家发现在炔胺的氮原子引入一个吸电子基团 (EWG), 即增加了炔胺的稳定性, 又赋予了其特殊的反 应活性, 使其稳定性和反应活性达到很好的平衡, 这类 特殊的炔胺被命名为炔酰胺 (Scheme 2) ${ }^{[3]}$. 同时, EWG 既可以作为定向取代基, 也可以作为手性辅助基团合成 手性炔酰胺, 有力地促进了不对称化学的发展 ${ }^{[4]}$. 另外, 炔酰胺在加成反应 ${ }^{[5]}$ 、环化反应 ${ }^{[6]}$ 、环异构化反应 ${ }^{[7]}$ 、金
属催化的交叉偶联反应 ${ }^{[8]}$ 、自由基反应 ${ }^{[9]}$ 等反应中也发 挥着极其重要的作用. 炔酰胺稳定性好, 大多数可以保 存数年而不变质, 并且易于纯化和后处理. 炔酰胺突出 的特性吸引了很多科学家的关注, 这使炔酰胺化学在近 二十年得到快速发展 ${ }^{[10]}$, 因此炔酰胺合成方法也越来 越受到人们重视. 本文将系统综述炔酰胺合成方法的研

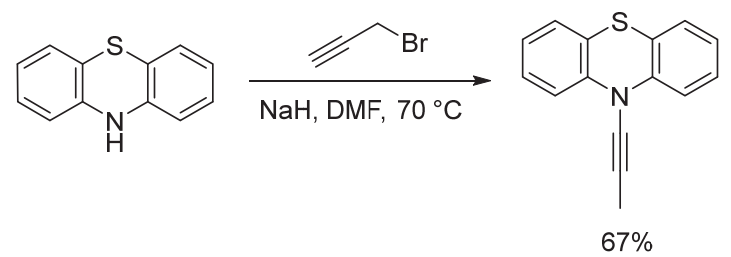

图式 1 炔胺的首次合成

Scheme 1 The first synthesis of ynamine

* Corresponding author. E-mail: zhaojf@jxnu.edu.cn

Received July 24. 2021; revised September 1, 2021; published online September 6, 2021.

Project supported by the National Natural Science Foundation of China (Nos. 21778025, 91853114).

国家自然科学基金(Nos. 21778025, 91853114 )资助项目. 


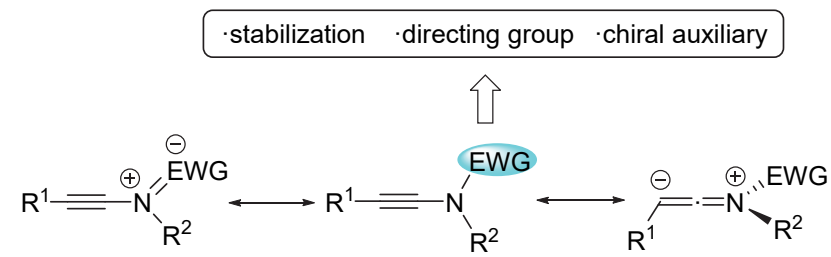

图式 2 炔酰胺的结构

Scheme 2 Structure of ynamides

究进展, 从而启发化学家发展出更多更加高效的炔酰胺 合成方法，并进一步推动炔酰胺化学的发展.

\section{1 卤代烯酰胺消除反应合成炔酰胺}

1972 年, Viehe 等 ${ }^{[11]}$ 通过消除反应实现了首例炔酰 胺的合成(Scheme 3). 反应分三步进行, 第一步是酰胺 与亚胺盐反应生成氯甲脒盐酸盐, 第二步是在碳酸氢钠 的水溶液条件下水解为烯酰胺, 最后在强碱 ${ }^{t} \mathrm{BuOK}$ 作用 下发生消除反应得到炔酰胺. 该方法的提出, 为炔酰胺 化学后续发展奠定了基础, 但是中间体制备条件苛刻且 步骤冗长, 底物普适性较差.

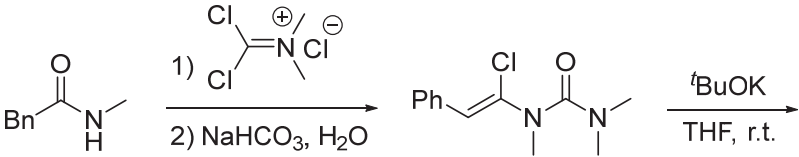

$$
\begin{aligned}
& \mathrm{Ph}_{\mathrm{l}} \mathrm{N}_{\mathrm{N}^{\prime}}^{\mathrm{O}}
\end{aligned}
$$

图式 3 炔酰胺的首次合成

Scheme 3 The first synthesis of ynamide

1994 年, Zemlicka 等 ${ }^{[12]}$ 成功实现了嘌呤和嘧啶类炔 酰胺的合成(Scheme 4). 胸腺嘧啶和胞嘧啶与四氯乙烯 在 $\mathrm{NaH}$ 为碱的条件下加热至 $60{ }^{\circ} \mathrm{C}$, 以 $25 \%$ 的收率得到 三氯烯酰胺, 然后在 $-70{ }^{\circ} \mathrm{C}$ 和 ${ }^{n} \mathrm{BuLi}$ 条件下以 $34 \%$ $51 \%$ 收率得到端炔酰胺. 尽管反应收率较低, 但开启了 端炔酰胺合成的先河.

2001 年, Hsung 等 ${ }^{[1]}$ 拓展了 Viehe 小组的方法, 烯酰 胺通过与 $N$-溴代丁二酰亚胺(NBS)或者液澳 $\left(\mathrm{Br}_{2}\right)$ 反应生 成溴代烯酰胺, 继而在强碱 ${ }^{t} \mathrm{BuOK}$ 作用下发生消除反应 得到炔酰胺(Scheme 5). 该方法成功地实现了基于吡咯 烷酮、噁唑烷酮和咪唑烷酮等结构的炔酰胺的合成.

2006 年, Brückner 等 ${ }^{[13]}$ 报道了以甲酰胺为原料制备 炔酰胺的方法 (Scheme 6). 首先在 $\mathrm{PPh}_{3} 、 \mathrm{CCl}_{4}$ 的条件下, 甲酰胺转化为中间体 $\beta, \beta$-二氯烯酰胺, 随后在 $-78{ }^{\circ} \mathrm{C}$ 加 入 ${ }^{n} \mathrm{BuLi}$, 并在一 $30{ }^{\circ} \mathrm{C}$ 下用无水甲醇淬灭反应得到端炔 酰胺 (method D). 但是制备甲酰胺路线如 methods A, B 和 $\mathrm{C}$, 不同的底物需要不同的条件, 整个过程过于繁琐, 在一定程度上限制了该方法的应用性. 在 Brückner 小组

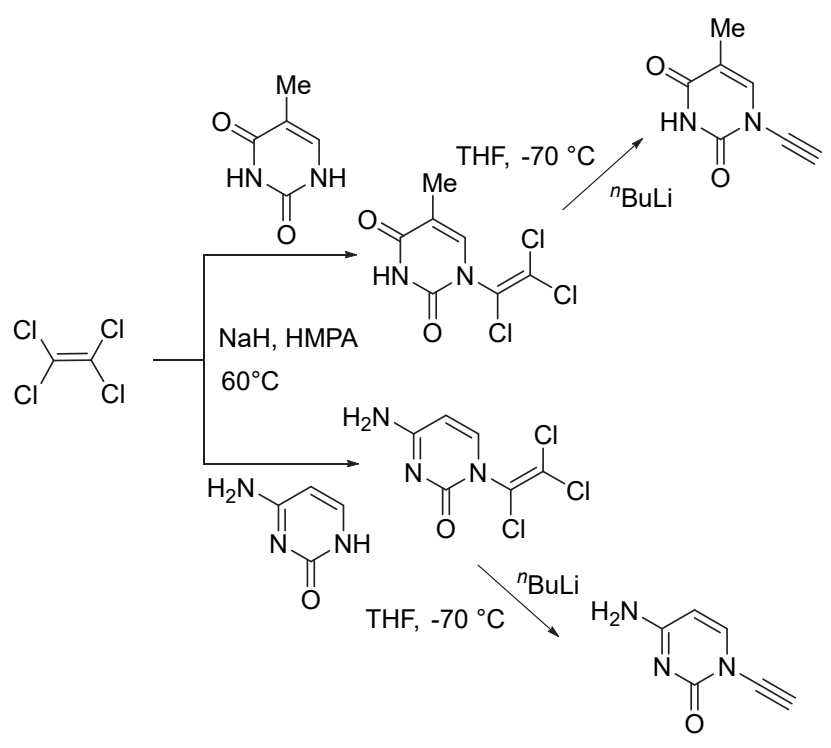

图式 4 端炔酰胺的首次合成

Scheme 4 The first synthesis of terminal ynamides

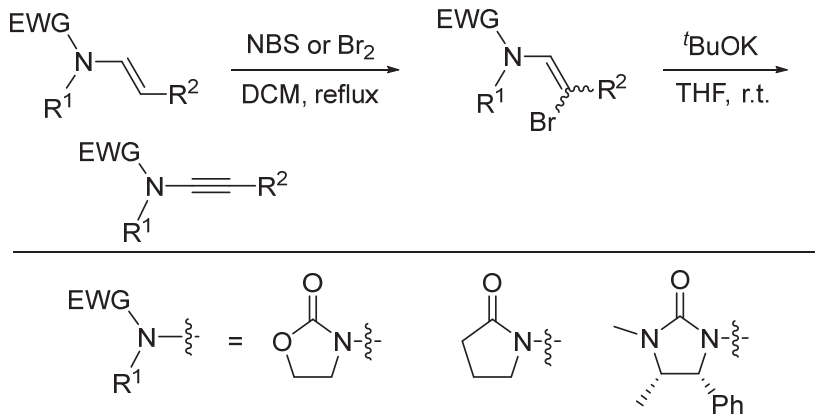

图式 5 消除反应合成炔酰胺

Scheme 5 Synthesis of ynamides via elimination reaction

的工作基础之上, Saá 小组 ${ }^{[14]}$ 利用 Negishi 偶联反应, 通 过 $\beta, \beta$-二氯烯酰胺形成中间体炔基锌试剂，随即加入钯 催化剂、膦配体以及芳基碘化合物，可以合成一系列炔 酰胺. Negishi 偶联反应的使用, 为当时内炔酰胺的合成 提供了一种新方法(method E). 不久, Saá 小组 ${ }^{[15]}$ 发展了 另一种更加简便的炔酰胺合成方法. 他们发现, $\beta, \beta$-二 氯烯酰胺在强碱 ${ }^{n} \mathrm{BuLi}$ 作用下形成中间体炔基锂，随即 炔基锂可以与各种亲电试剂反应得到相应炔酰胺 $(\operatorname{method} \mathrm{F})$.

2015 年, Anderson 小组 ${ }^{[16]}$ 以三氯乙烯为原料报道合 成炔酰胺的方法(Scheme 7a). 反应分两步进行: 第一步 是磺酰胺在碱性条件下与三氯乙烯反应生成 1,2-二氯烯 酰胺，第二步是在苯基锂作用下形成炔基锂中间体后加 入各种亲电试剂形成炔酰胺. 2019 年, 该小组 ${ }^{[17]}$ 还发现 1,2-二氯烯酰胺在双(三甲基硅基)氨基锂(LiHMDS)作用 下形成氯代炔酰胺中间体, 再在铜催化条件下可以与各 种金属试剂反应生成一系列炔酰胺(Scheme 7b).

Cossy 小组 ${ }^{[18]}$ 报道了一种利用 Suzuki-Miyaura 偶联 


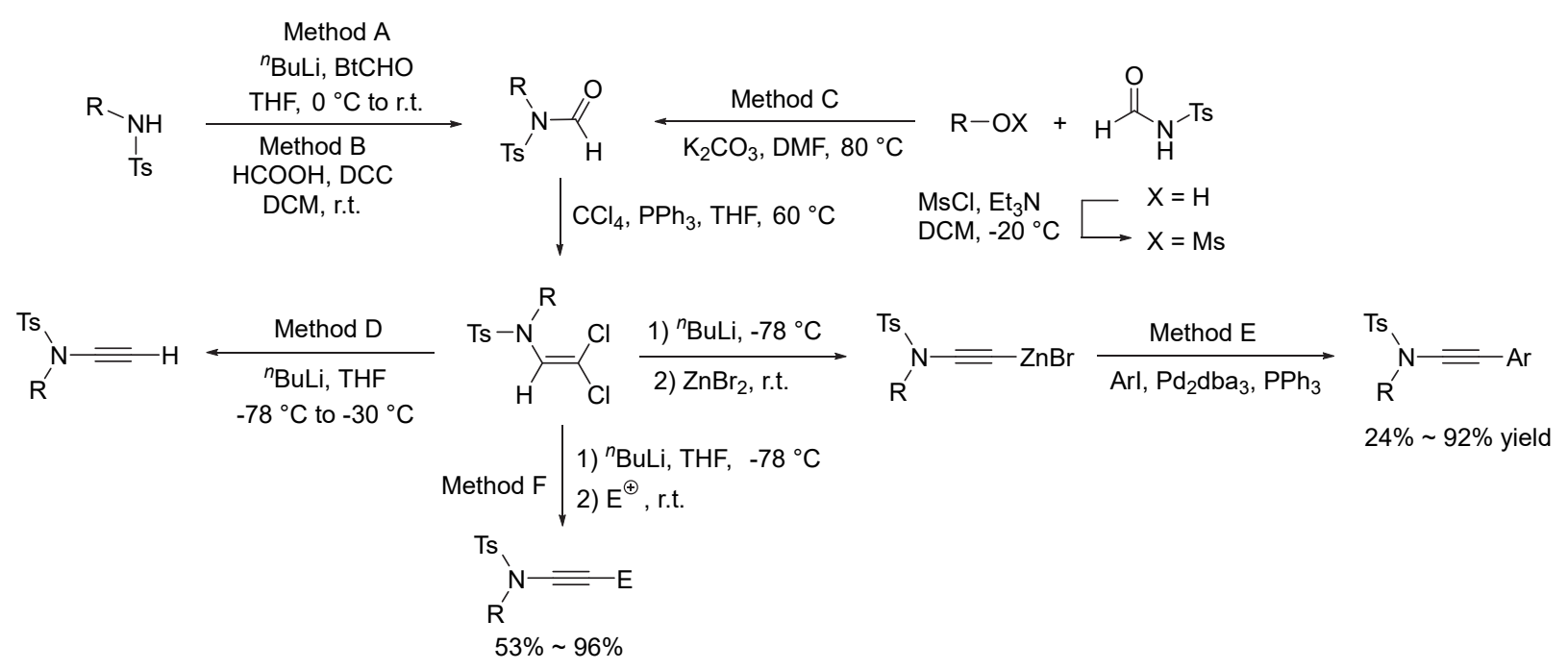

图式 6 以甲酰胺为原料合成炔酰胺

Scheme 6 Synthesis of ynamides with formamides

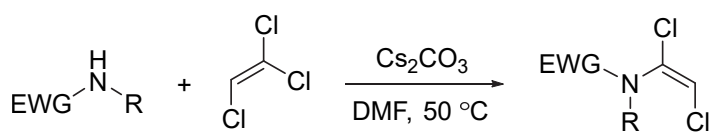

a) EWG $\mathrm{N}=\mathrm{R}^{1}$

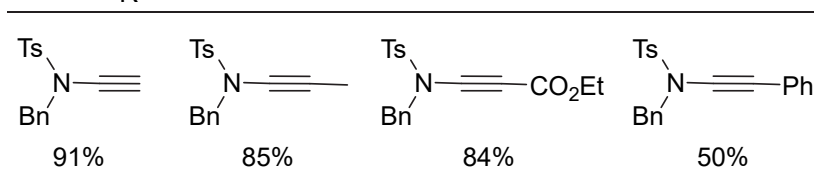

b)

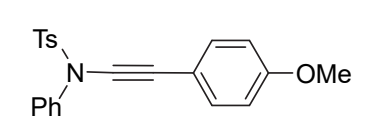

$83 \%$

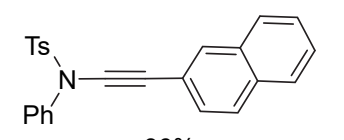

$86 \%$<smiles>[13F]N(C#Cc1ccccc1Br)c1ccccc1</smiles>

$76 \%$

图式 7 三氯乙烯为原料合成炔酰胺

Scheme 7 Synthesis of ynamides with trichloroethene

反应合成各种内炔酰胺的方法(Scheme 8). 利用 Brückner 工作中的方法先合成中间体二氯烯酰胺, 然后和嗍酸发 生 Suzuki-Miyaura 偶联反应得到 $(E)-\beta$-氯代烯酰胺, 最 后在双(三甲基硅基)胺基锂或者氢氧化钠/四丁基硫酸
氢胺条件下发生消除反应生成炔酰胺.

综上所述: 以卤代烯酰胺为原料, 通过消除反应合 成炔酰胺，为炔酰胺的合成打开了新的局面，但此类反 应需在强碱条件下进行，同时反应的底物范围也受到限 制.

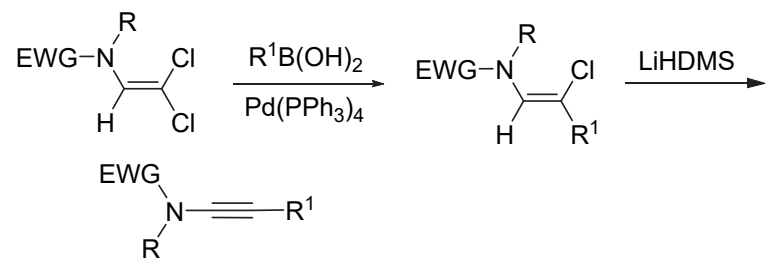

图式 8 Suzuki-Miyaura 偶联反应合成炔酰胺

Scheme 8 Synthesis of ynamides by Suzuki-Miyaura coupling

\section{$2 N$-亲核试剂与炔烃及其衍生物合成炔酰胺}

\section{$2.1 N$-亲核试剂与高价炔碘盐合成炔酰胺}

继 Stang 小组 ${ }^{[19]}$ 报道了使用氨基锂试剂与芳基炔碘 盐合成炔酰胺的方法之后(Scheme 9), Witulski 小组 ${ }^{[20]}$ 详 细地报道了炔碘盐合成炔酰胺的机理. 他们认为机理可 能是 $\mathrm{N}$ 亲核试剂先进攻炔烃的 $\beta$ 位发生亲核加成反应产 生烯基卡宾，再经过 1,2-迁移形成炔酰胺(Scheme 10). 同时他们讨论了酰胺的空间位阻效应, 发现具有 $\alpha$-支链 的酰胺收率偏低, 推测空间位阻可能会影响酰胺对炔烃 的亲核加成. 硅基保护的炔酰胺在四丁基氟化铵 (TBAF)条件下, 去硅基化后可以高收率得到端炔酰胺.

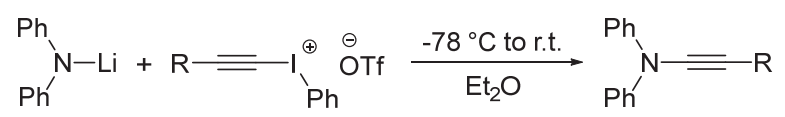

图式 9 炔碘盐为原料合成炔酰胺

Scheme 9 Synthesis of ynamides with alkynyl iodonium salts 


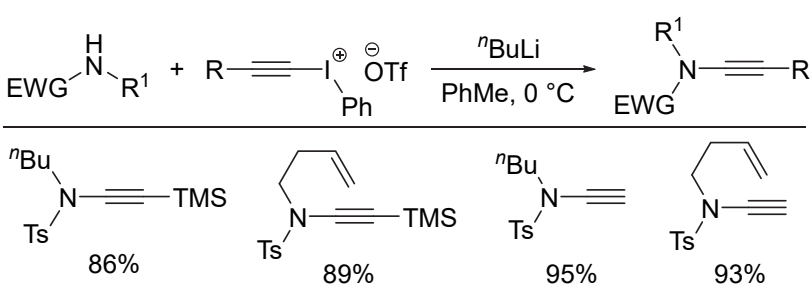

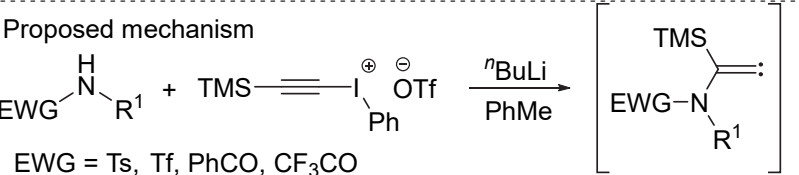

$$
\begin{aligned}
& \mathrm{R}^{1}=\text { alkyl, } \mathrm{Bn}
\end{aligned}
$$

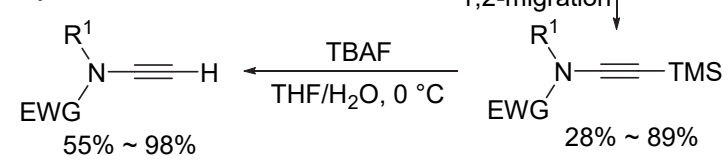

图式 10 炔碘盐合成炔酰胺以及其可能反应机理

Scheme 10 Synthesis of ynamides with alkynyl iodonium salt and the possible mechanism

Witulski 等 ${ }^{[21]}$ 采取 Stang 小组的策略合成出一系列 含有烯烃以及炔烃的炔酰胺, 促进了炔酰胺参与的加成 以及环加成反应发展(Scheme 11). Rainier 小组 ${ }^{[22]}$ 以炔碘 盐、炔基锂试剂和氮杂环丙烷试剂为原料, 获得含有不 同炔烃的炔酰胺(Scheme 12). 氮杂环丙烷试剂在炔基 锂亲核作用下开环, 与高价炔碘盐反应得到最终产物炔 酰胺.

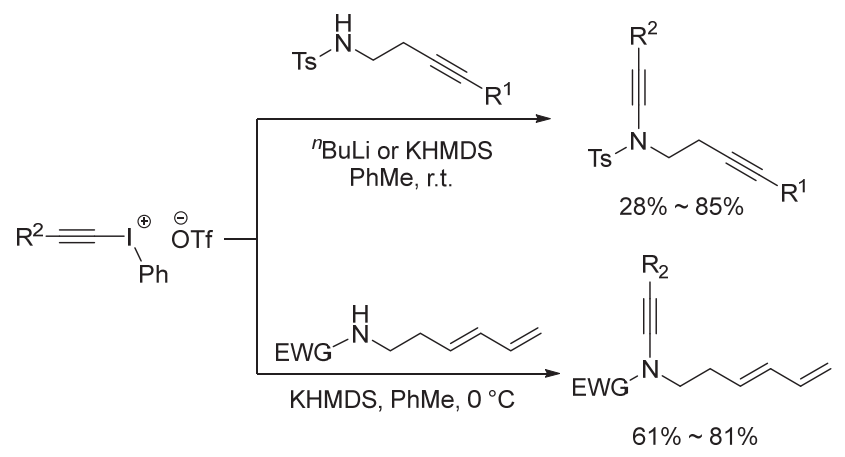

图式 11 高度官能团化的炔酰胺合成

Scheme 11 Synthesis of highly functionalized ynamides

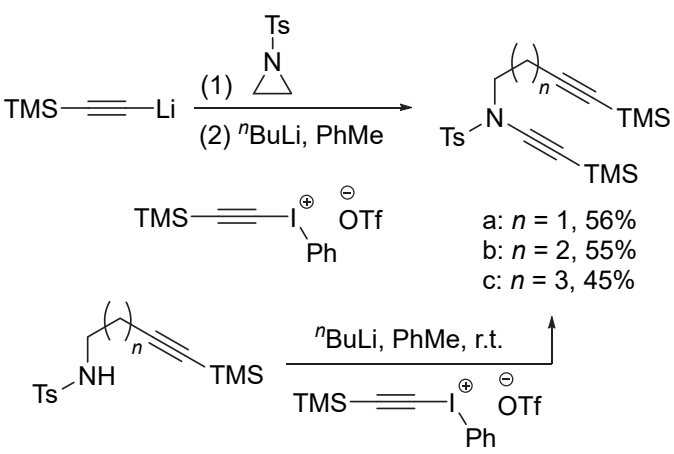

图式 12 含有炔基的炔酰胺合成

Scheme 12 Synthesis of ynamides containing an alkynyl group
1996 年, Feldman 等[23]使用亲电性较强的高价炔碘 盐, 首次成功合成了手性炔酰胺(Scheme 13). 不久, Witulski 小组 ${ }^{[24]}$ 利用同样的策略合成出具有手性 $\alpha$-支链 炔酰胺(Scheme 14), 且只有当 $R^{2}=H$ 时, 反应效果最 好; 当 $\mathrm{R}^{2}=$ 三甲基硅烷(TMS)时，反应不发生. 作者推 测可能是位阻效应导致，所得结果与他之前工作结果一 致(当酰胺 $\alpha$ 位位阻较大时, 合成手性炔酰胺效果较差).

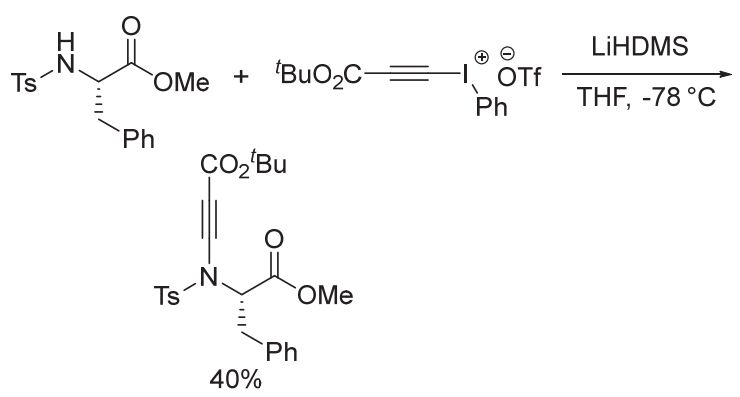

图式 13 手性炔酰胺的首次合成

Scheme 13 The first synthesis of chiral ynamides

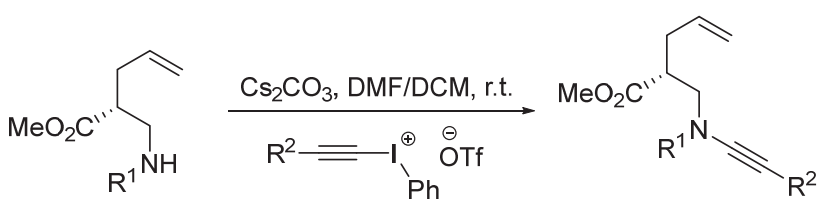
$\mathrm{R}^{1}=\mathrm{Ts}, \mathrm{Ns}, \mathrm{Tf}, 2$-pyridyl- $\mathrm{SO}_{2}$

$R^{2}=H, 70 \% \sim 95 \%$ 4 examples $\mathrm{R}^{2}=\mathrm{TMS}, 0 \%$

图式 14 手性 $\alpha$-支链的炔酰胺合成

Scheme 14 Synthesis of chiral $\alpha$-branched ynamides

综上所述, 21 世纪初, 高价炔碘盐作为炔烃前体合 成炔酰胺将炔酰胺化学向前推进了一步, 然而此类反应 大都需在强碱性条件下发生，且大部分的炔基碘盐不稳 定，原料的制备较为繁琐，一定程度限制了其应用. 为 克服以上缺点, Muñiz 等 ${ }^{[25]}$ 通过末端炔烃与高价碘试剂 $\mathrm{PhI}(\mathrm{OAc}) \mathrm{NTs}_{2}$ 的反应开发了一种新的形成内炔酰胺的 方法(Scheme 15). 该方法的优点是不需要使用过渡金 属, 通过末端炔烃与高价碘试剂 $\mathrm{PhI}(\mathrm{OAc}) \mathrm{NTs}_{2}$ 一步反 应制备内炔酰胺，操作步骤简单，但是该方法仅适用于 芳基炔烃. 2019 年，李栋小组 ${ }^{[26]}$ 报道了二苯磺酰亚胺与 各种芳基炔烃在醋酸碘苯氧化剂存在下通过氧化偶联 反应生成内炔酰胺的方法(Scheme 16). 值得注意的是, 该方法在醋酸碘苯条件下生成内炔酰胺，但是在无醋酸 碘苯条件下生成烯酰胺类化合物.

2019 年, Itoh 和 Tada 小组 ${ }^{[27]}$ 发现了一种乙炔基苯并 恶唑啉酮(EBX)-乙腈络合物，并将其作为炔烃前体合成 出一系列炔酰胺(Scheme 17). 反应体系中, 不但磺酰胺 反应良好，以中等偏高收率获得相应炔酰胺，一些氨基 酸以及多肽衍生物也可以兼容. 


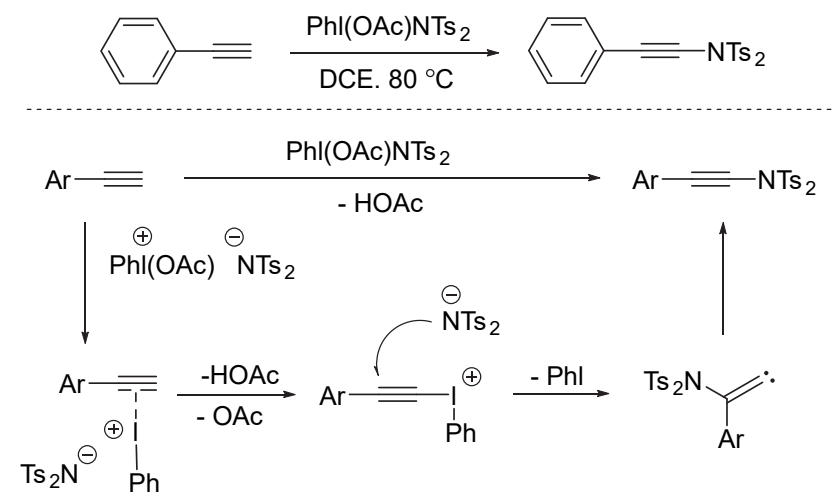

图式 15 无过渡金属合成炔酰胺

Scheme 15 Metal-free synthesis of ynamides

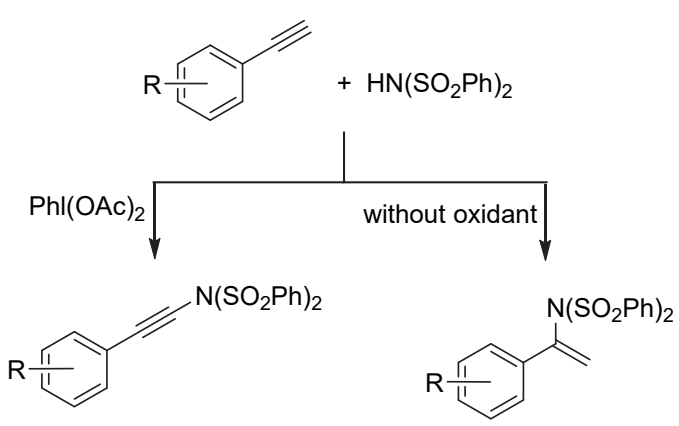

图式 16 醋酸碘苯作为氧化剂合成炔酰胺

Scheme 16 Synthesis of ynamides with PIDA as oxidant

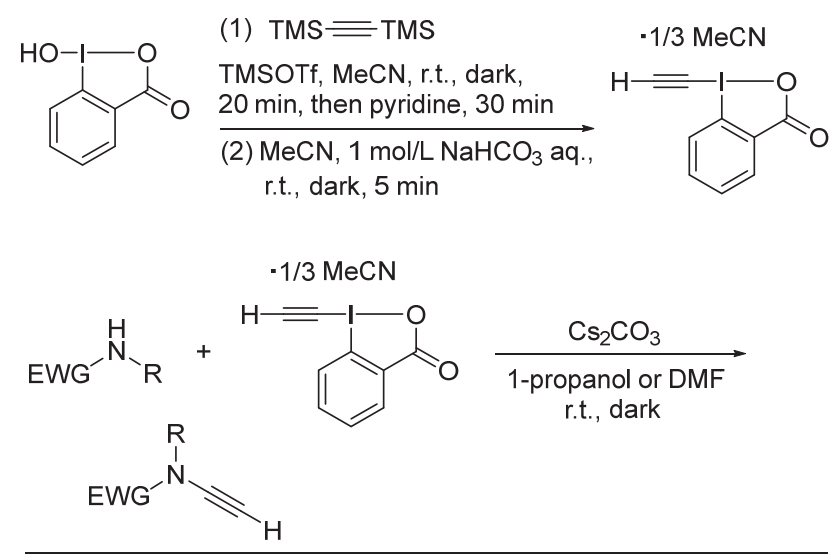<smiles>C#CN(C)P</smiles>

$90 \%$<smiles>C#CN(F)C(Cc1ccccc1)C(=O)OC</smiles><smiles>C#CN([18F])CC=C</smiles>

$74 \%$<smiles>C#CN1[13CH]C(C)CC1C(=O)N[C@H](C(=O)OC)C(C)C</smiles>

图式 $17 \mathrm{EBX}$-乙腈络合物作为原料合成炔酰胺 Scheme 17 Synthesis of ynamides with EBX-MeCN complex
最近, Itoh 和 Tada 小组利用高价炔基碘试剂与氨基 酸为原料合成了一系列特殊的炔酰胺(Scheme 18) ${ }^{[28]}$. 富电子双齿联吡啶配体(4,4'-二甲氧基-2,2'-联吡啶)和质 子性溶剂 $(\mathrm{EtOH})$ 是促使反应发生的关键因素, 可能与 $\mathrm{CuI}$ 形成了活性铜物种，并抑制自身偶联副产物的产生. 值得一提的是亮氨酸衍生的炔酰胺通过氢化反应、水合 反应以及偶联反应可以得到独特的氨基酸衍生物.

$$
\mathrm{EWG}^{-\mathrm{N}_{-\mathrm{R}^{1}}}
$$<smiles></smiles><smiles>CCCCC#CN([13CH3])C(CC(C)C)C(=O)OCC</smiles>

Application of leucine-derived ynamide:

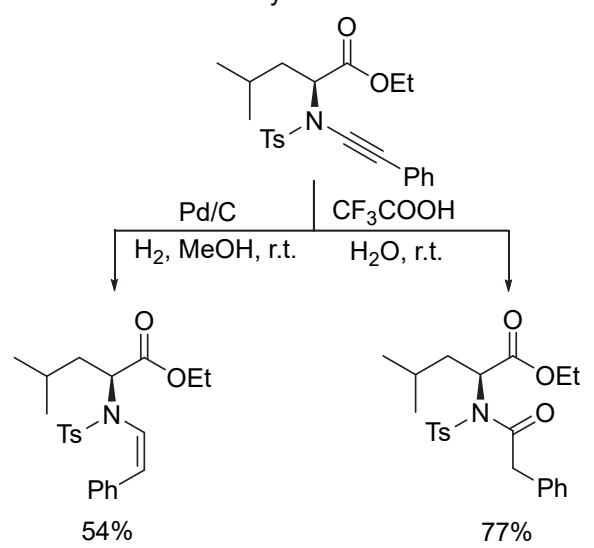

图式 18 高价炔基碘为原料合成炔酰胺以及亮氨酸衍生炔酰 胺的应用

Scheme 18 Synthesis of ynamides with hypervalent alkynyl iodine reagents and application of leucine-derived ynamide

\section{$2.2 N$-亲核试剂与卤代炔烃合成炔酰胺}

2003 年, Hsung 等 ${ }^{[29]}$ 首次发现铜催化合成内炔酰胺 方法(Scheme 19). 反应中以酰胺和炔溴为原料, $\mathrm{K}_{3} \mathrm{PO}_{4}$ 为碱，只需要 $5 \mathrm{~mol} \% \mathrm{CuCN}$ 或者 $\mathrm{CuI}$ 催化剂, $10 \mathrm{~mol} \%$ $N, N$-二甲基乙二胺(DMEDA)作为配体，在甲苯回流下 反应即可高效地得到炔酰胺. 该方法原子经济性高, 并 

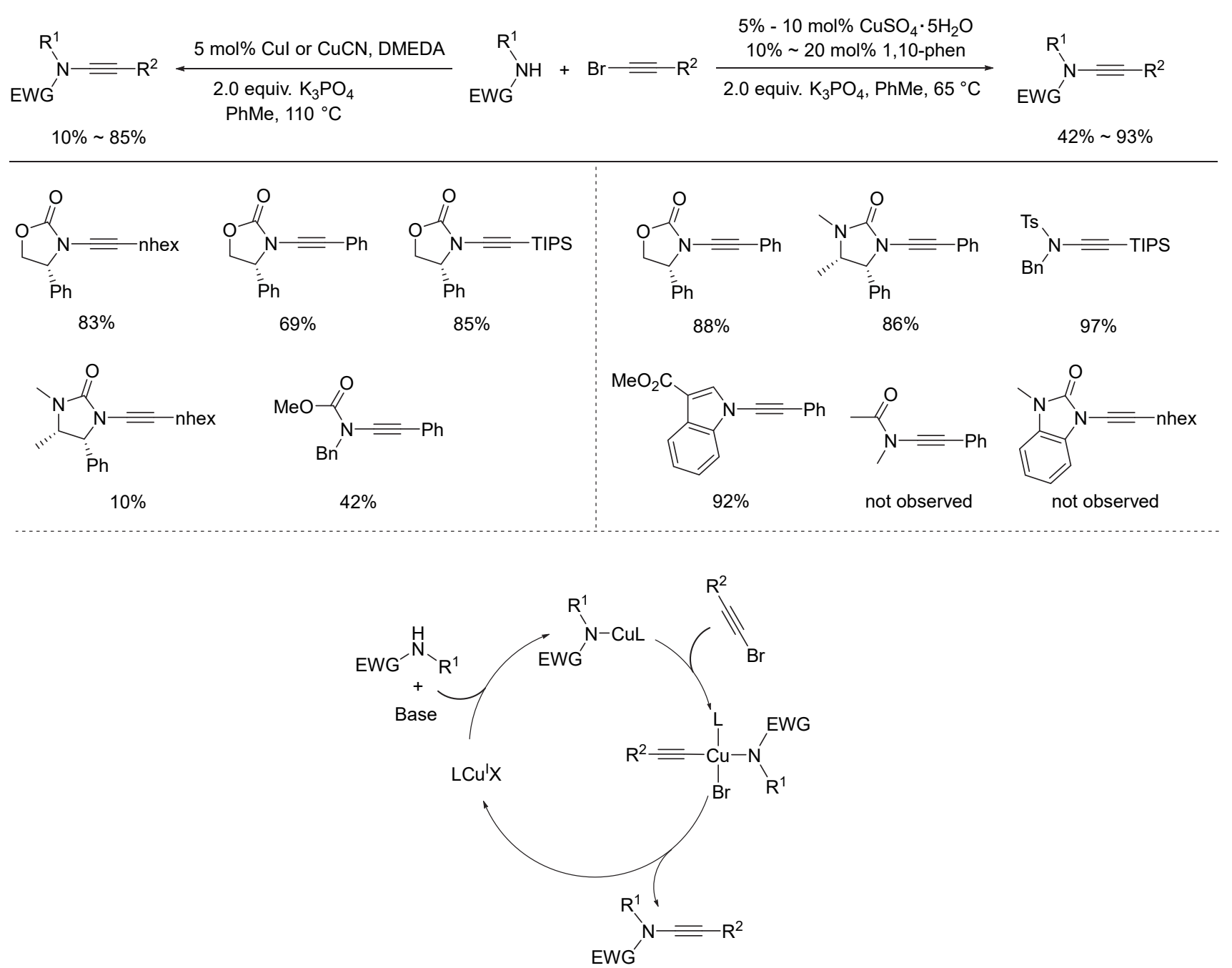

图式 19 铜催化首次合成内炔酰胺以及可能反应机理

Scheme 19 The first synthesis of internal ynamides by copper catalysis and proposed mechanism

且底物适用性广，包括噁唑烷酮以及内酰胺都可以以较 好收率得到相应炔酰胺. 在机理方面, 作者推测在碱性 条件下, 酰胺可能先与铜催化剂形成铜物种, 再与炔溴 发生氧化加成得到炔基铜物种，最后还原消除得到产物 炔酰胺. 2004 年 Hsung 小组 ${ }^{[30]}$ 发现了一种更好的炔酰胺 合成方法. 与之前 $\mathrm{CuCN}$ 体系相比，除 $\mathrm{CuSO}_{4} \cdot 5 \mathrm{H}_{2} \mathrm{O}$ 价 格更便宜之外，还具有以下优势：(1)新体系底物适用性 更为广泛，如效果不好的咪唑烷酮和磺酰胺类化合物， 在新体系中也能得到较好收率; (2)炔酰胺的制备规模甚 至可以达到 $100 \mathrm{mmol}^{[31]}$. 这是当时以过渡金属铜为催 化剂合成炔酰胺最经济高效的制备方法. 但是, Hsung 体系中一些简单的炔酰胺并不适用 ${ }^{[32]}$.

2003 年, Danheiser 等 ${ }^{[33]}$ 报道了铜介导酰胺与炔溴 直接偶联合成炔酰胺的方法(Scheme 20). 酰胺在等物 质的量的 $\mathrm{CuI}$ 作用下形成铜试剂, 随后与炔溴加成形成 炔酰胺. 该反应的优点是反应在室温下进行, 反应条件 温和, 可以较高收率得到之前不易合成的酰胺类内炔酰
胺，该方法还可用于大量制备 ${ }^{[34]}$.

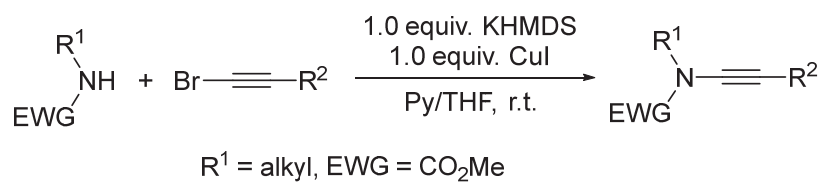

图式 20 铜介导的炔酰胺合成

Scheme 20 Cu-mediated synthesis of ynamides

基于上述反应，Pale 小组 ${ }^{[35]}$ 继续研究了炔基溴与二 级酰胺制备内炔酰胺的反应. 该团队改变了以往铜催化 合成内炔酰胺反应的条件，提出了一种铜一沸石(USY) 催化合成内炔酰胺的方法(Scheme 21). 该方法的优点 是铜一沸石催化剂可以回收和重复使用至少 5 次，且没 有显著的失活，并且磺胺类化合物、噁唑烷酮类化合物 和吲哚类化合物都可以较高收率得到相应的内炔酰胺.

2009 年, 张玉红小组 ${ }^{[36]}$ 首次使用铁盐为催化剂合 成内炔酰胺. 该方法以酰胺类化合物与炔基溴为原料, 


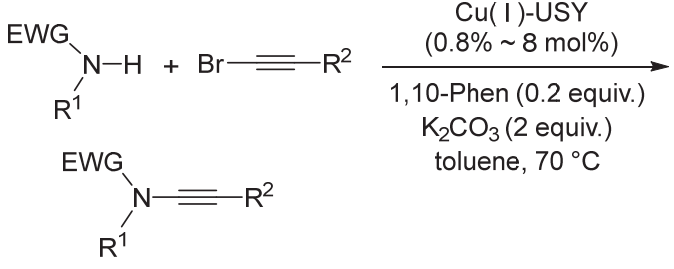

图式 21 铜-沸石(USY)催化制备内炔酰胺

Scheme 21 Synthesis of ynamides by copper zeolite (USY) catalysis

六水合氯化铁为催化剂, DMEDA 为配体, $\mathrm{K}_{2} \mathrm{CO}_{3}$ 为碱, 甲苯为溶剂的情况下, 可以合成出理想的内炔酰胺 (Scheme 22). 该方法的优点是使用价格低廉、易于处理 且对环境无害的六水合氯化铁为催化剂, 更加绿色环 保.

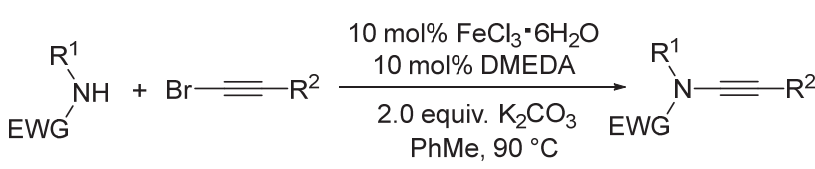

$$
\begin{aligned}
& \mathrm{R}^{2}=\text { Aryl, Alkyl, TIPS, } \mathrm{CH}_{2} \mathrm{OH}
\end{aligned}
$$

图式 22 首次使用铁催化剂合成内炔酰胺

Scheme 22 The first synthesis of ynamide by Fe catalysis

2019 年, 黄申林小组 ${ }^{[37]}$ 采用松香基表面活性剂 APGS-550-M 胶束催化策略合成内炔酰胺(Scheme 23). 该方法实现在水中合成炔酰胺. 该方法的优点是表面活 性剂 APGS-550-M 廉价易得, 容易从天然丰富的生物 质、树脂酸中制备; 以水代替有机溶剂, 使得合成方法 更加绿色环保. 且此反应可以放大为克级规模.

$$
\begin{gathered}
\underset{\mathrm{R}^{2^{\prime}}}{\mathrm{NH}}+\mathrm{Br}=\mathrm{R}^{1} \frac{[\mathrm{Cu}] \text {, ligand, } \mathrm{K}_{2} \mathrm{CO}_{3}}{\begin{array}{c}
2 \mathrm{wt} \% \mathrm{APGS}-550-\mathrm{M} / \mathrm{H}_{2} \mathrm{O} \\
36 \text { examples, up to } 98 \% \text { yield }
\end{array}} \\
\text { no organic solvent, recycable, gram-scale, low } \mathrm{E} \text { factor }
\end{gathered}
$$

图式 23 APGS-550-M 催化合成炔酰胺

Scheme 23 Synthesis of ynamides by APGS-550-M catalysis

2011 年, Hsung 小组 ${ }^{[38]}$ 首次以磷酰胺和炔溴为原料 合成含 $N$-磷酰基的炔酰胺(Scheme 24). 2014 年 Bolm 小 组 ${ }^{[39]}$ 报道亚砜亚胺和炔溴在 $\mathrm{Cu}(\mathrm{OAc})_{2}$ 催化条件下合成 炔酰胺的方法(Scheme 25), 该方法条件温和, 底物拓展 范围宽广. 2016 年, Magnie 等 ${ }^{[40]}$ 报道了以 $S$-全氟亚砜亚 胺和炔溴在碘化铜催化下生成炔酰胺的方法(Scheme 26).

硅基保护的炔烃与酰胺的偶联反应已成为合成端 炔酰胺通用的方法 ${ }^{[29]}$ (Scheme 27). 在 $110 \sim 150{ }^{\circ} \mathrm{C}$ 下, 以 $\mathrm{CuCN}$ 作为催化剂, $\mathrm{DMEDA}$ 作为配体, $\mathrm{K}_{3} \mathrm{PO}_{4}$ 作
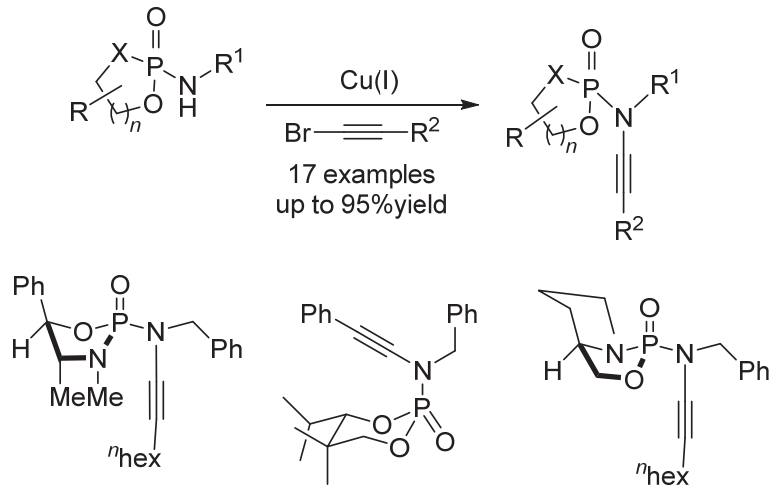

图式 $24 N$-磷酰基的炔酰胺合成

Scheme 24 Synthesis of $N$-phosphoryl ynamides

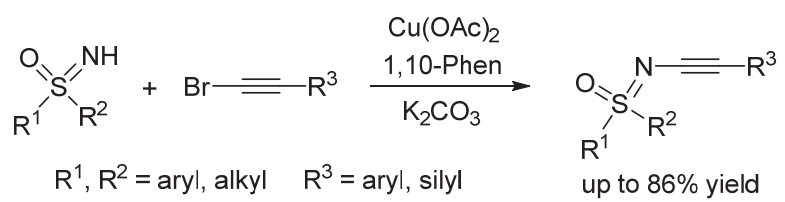

图式 $25 N$-炔基亚砜亚胺合成

Scheme 25 Synthesis of $N$-alkynyl sulfoximines

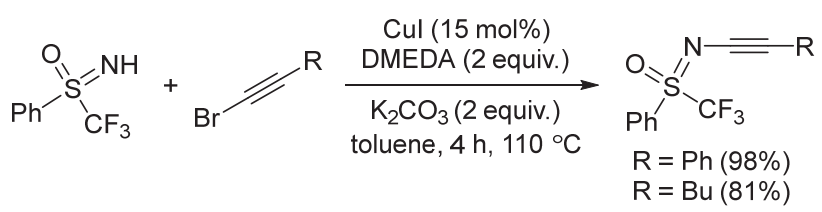

图式 $26 S$-全氟炔基亚砜亚胺合成

Scheme 26 Synthesis of $S$-perfluoro alkynyl sulfoximines

为碱, 较高收率得到噁唑烷酮类炔酰胺, 对于普通酰胺 以及氨基甲酰胺，收率较低. 以三异丙基硅烷(TIPS)保 护的炔酰胺经过脱硅化处理即可得到相应的端炔酰胺.

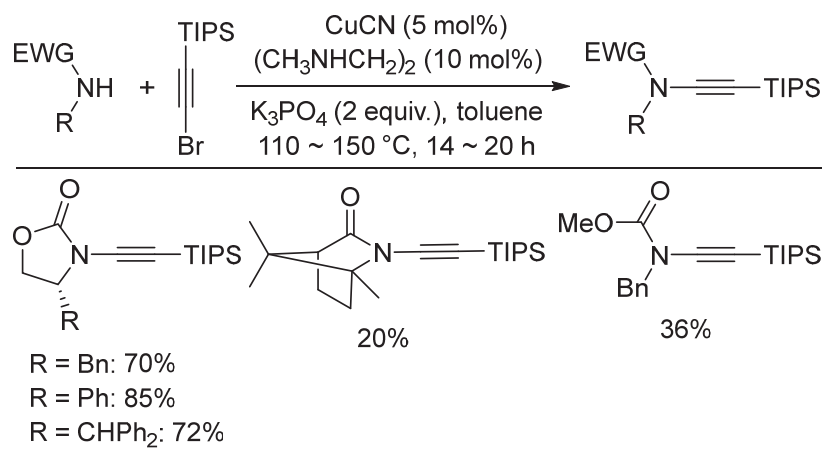

图式 $27 \mathrm{CuCN}$ 催化噁坐烷酮和氨基甲酸酯类炔酰胺合成 Scheme 27 Synthesis of ynamides containing oxazolidinone or carbamate by $\mathrm{CuCN}$ catalysis

不久, Urabe 等 ${ }^{[41]}$ 以 $5 \mathrm{~mol} \% \mathrm{CuI}$ 为催化剂, TIPS 保 护的炔溴和二级酰胺为原料, 可以获得一系列 TIPS 保 护的炔酰胺, 后续通过 TBAF 脱硅化处理可以得到相应 的端炔酰胺(Scheme 28). 


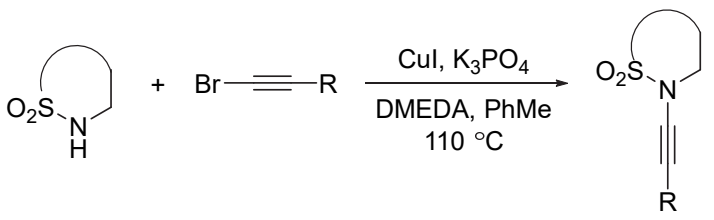<smiles>[R]C1c2ccccc2S(=O)(=O)N1C#C[In]S</smiles><smiles>CC1(C)C2CCCC1C1(CCCC1)C2</smiles><smiles>COCC(c1ccccc1)N([13CH3])C#CS[InH]</smiles>

$58 \%$

$\mathrm{R}=\mathrm{Me} ; 71 \%$

$\mathrm{R}={ }^{n} \mathrm{Bu}: 66 \%$

$\mathrm{R}=\mathrm{Bu} ; 71 \%$

$23 \%$

图式 $28 \mathrm{CuI}$ 催化亚磺酰胺的 $N$-炔基化反应

Scheme 28 CuI catalyzed $N$-alkynylation of sulphonamides

Danheiser 等 ${ }^{[42]}$ 报道了一种在温和条件下分两步合 成炔酰胺的方法(Scheme 29): 第一步，氨基甲酸酯在化 学计量的 $\mathrm{CuI}$ 和双三甲基硅烷基氨基钾(KHMDS)条件 下生成铜络合物, 然后再与炔溴反应得到炔酰胺. 该方 法在室温下即可高收率得到一系列炔酰胺.

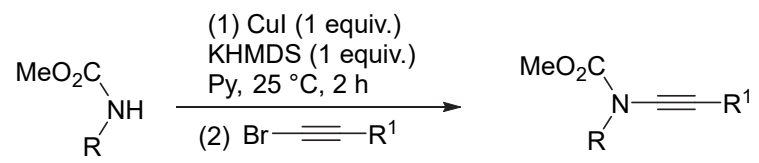

$\mathrm{R}=\mathrm{CH}_{2} \mathrm{CH}_{2} \mathrm{Ph}, \mathrm{R}^{1}=\mathrm{SiMe}_{3}: 56 \% ; \mathrm{R}=\mathrm{Bn}, \mathrm{R}^{1}=\mathrm{Si}\left({ }^{i} \mathrm{Pr}\right)_{3}: 74 \%$;

$\mathrm{R}=\mathrm{Bn}, \mathrm{R}^{1}=\mathrm{H}: 81 \%$

图式 29 铜介导的氨基甲酸酯的 $N$-炔基化反应

Scheme 29 Copper-mediated $N$-alkynylation of carbamates

Hsung ${ }^{[30 b, 34]}$ 、Tam ${ }^{[43]}$ 等对上述反应条件进行了改进, 用硫酸铜、二羧酸硫苯铜、碘化亚铜等作催化剂, 1,10菲罗啉或 DMEDA 为配体, 降低了反应温度, 提高了反 应产率(Scheme 30). 炔酰胺产率可达 97\%, 反应可达 $100 \mathrm{mmol}$ 的规模.

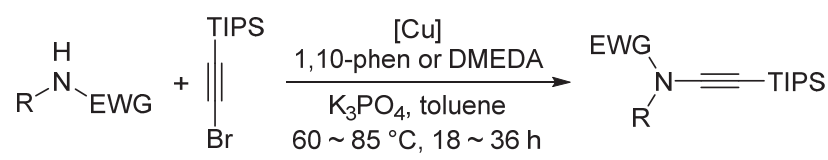

图式 30 铜盐和 1,10-菲罗啉体系下合成炔酰胺

Scheme 30 Synthesis of ynamides with the combination of $\mathrm{Cu}$-salt and 1,10-phen

2015 年, Trolez 等 ${ }^{[44]}$ 报道了多炔酰胺的合成. 借助 Hsung 和 Evano 合成炔酰胺的方法, 以炔溴为原料, 在 $\mathrm{K}_{2} \mathrm{CO}_{3} 、 \mathrm{CuSO}_{4}$ 和 1,10 -菲罗啉的条件下, 与磺酰胺反应 生成双炔酰胺和三炔酰胺(Scheme 31).

\section{$2.3 N$-亲核试剂与炔酸及其他炔烃衍生物合成炔酰胺}

2010 年，焦宁等 ${ }^{[45]}$ 提出了一种铜催化氧化脱羧
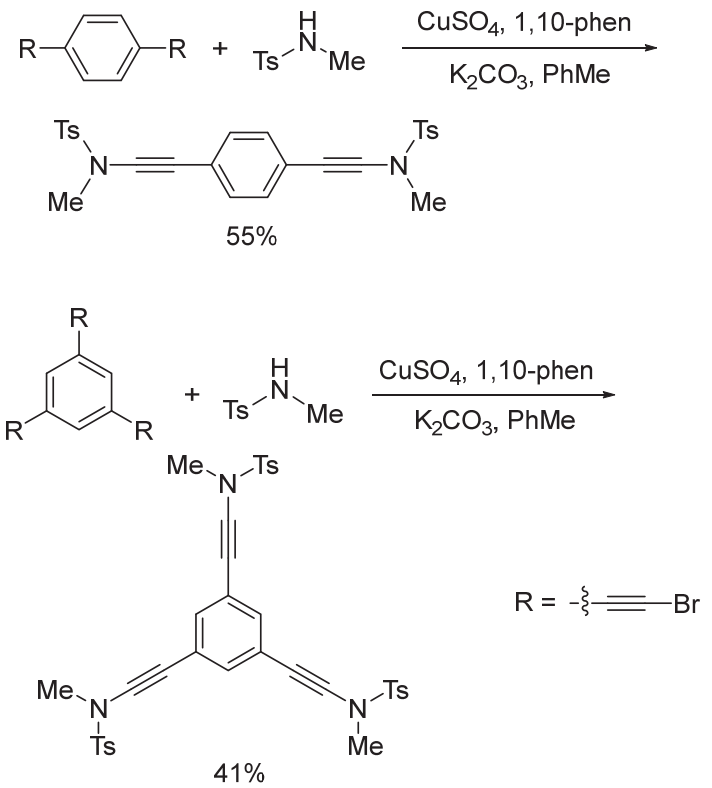

图式 $31 \mathrm{CuSO}_{4}$ 催化多炔酰胺合成

Scheme 31 Synthesis of multi-ynamides by $\mathrm{CuSO}_{4}$ catalysis

反应生成内炔酰胺的方法. 丙炔酸可以和噁唑烷酮、咪 唑烷酮、内酰胺、磺胺类和吲哚反应生成相应内炔酰胺 (Scheme 32). 丙炔酸作为炔烃的前体具有以下优点: (1) 反应在空气中进行，条件简单. (2)可以有效抑制二炔类 化合物的生成，并减少催化剂和亲核试剂的用量. (3)丙 炔酸衍生物容易获得，易于储存. (4)该反应的副产物只 有二氧化碳产生, 绿色环保. 不久, Bolm 小组 ${ }^{[46]}$ 利用相 同策略实现了 $N$-炔基亚砜亚胺的合成(Scheme 33).
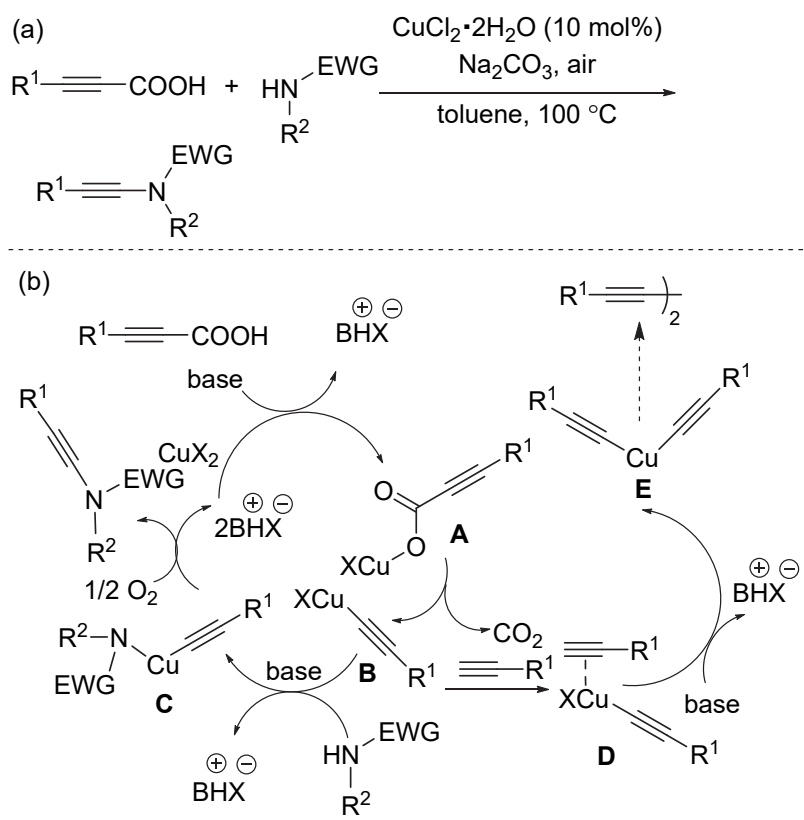

图式 32 丙烯酸的氧化氨化反应以及可能反应机理 Scheme 32 (a) Oxidative amidation and (b) proposed mechanism of propiolic acids 


$$
\begin{aligned}
& \begin{array}{c}
10 \mathrm{~mol} \% \mathrm{CuBr} \\
\mathrm{R}^{1}
\end{array} \mathrm{~S}^{\mathrm{N}} \mathrm{R}^{2}+\mathrm{HOOC}=\mathrm{R}^{3} \quad \begin{array}{c}
20 \mathrm{~mol} \% \mathrm{Py}_{\mathrm{K}} \mathrm{PO}_{4} \\
\mathrm{PhMe}, \text { air, } 80{ }^{\circ} \mathrm{C}, 16 \mathrm{~h}
\end{array} \\
& \text { O、 } N=R^{3} \\
& R^{1} R^{2}
\end{aligned}
$$
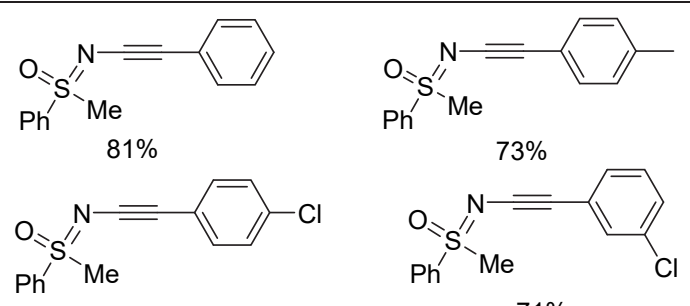

$82 \%$
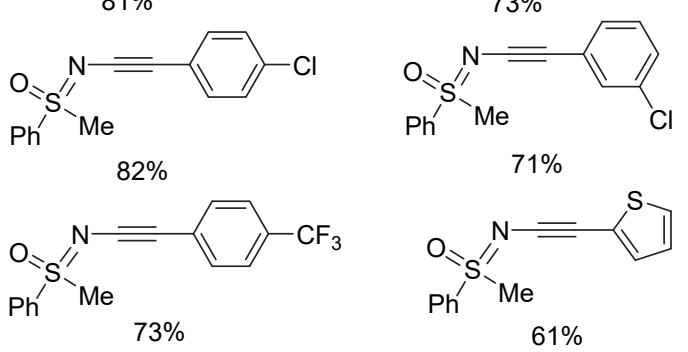

图式 33 氧化脱羧偶联合成 $N$-炔基亚砜亚胺

Scheme 33 Synthesis of $N$-alkynyl sulfoximines by oxidative decarboxylation coupling

上述合成炔酰胺的方法中大都需要高温以及过量 的碱. 并不满足原子经济性和环境友好的要求. Evano 小组 ${ }^{[47]}$ 报道了一种在室温条件下, 无需加碱合成炔酰 胺的方法(Scheme 34). 酰胺和炔基硼试剂在铜催化, 室 温下反应, 以较好收率得到炔酰胺. 这种方法底物适用 性广, 尤其是具有特殊官能团的炔酰胺, 例如羟基、双 键，但是炔基硼试剂的制备条件苛刻，一定程度限制了 此方法的应用.

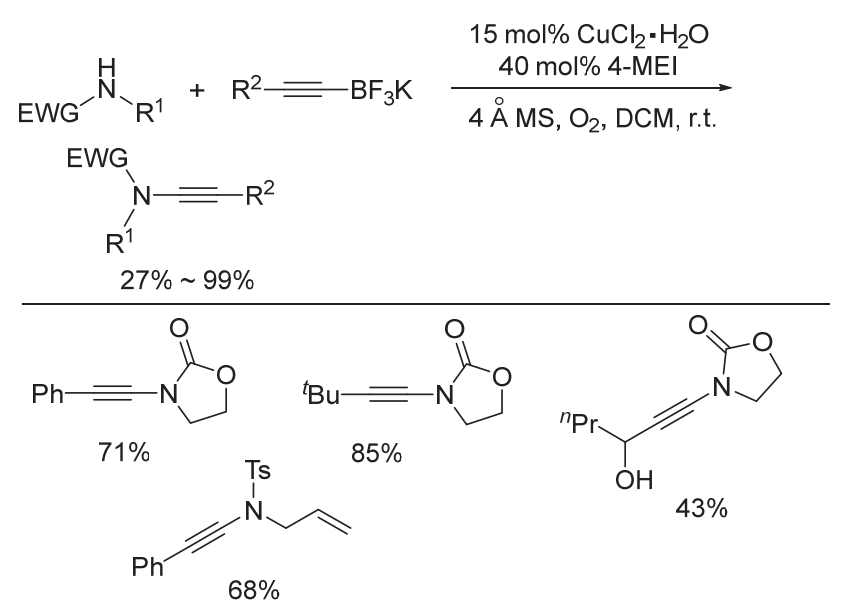

图式 34 炔基嗍试剂和酰胺合成炔酰胺

Scheme 34 Synthesis of ynamides with alkynylboron reagents and amides

2012 年, Evano 小组 ${ }^{[48]}$ 提出了一种炔基铜与酰胺通 过氧化偶联反应合成炔酰胺的方法(Scheme 35). 该方 法可分为两步：第一步为炔基铜试剂的制备. 炔烃与碘 化铜在氨水和乙醇的混合物或者含有 $\mathrm{K}_{2} \mathrm{CO}_{3}$ 的条件下
制备炔基铜试剂; 第二步为炔基铜试剂和二级酰胺反应 得到相应内炔酰胺. 该方法的优点是只需将二级酰胺、 炔基铜试剂和 TMEDA 置于乙腈中室温下搅拌, 即可以 良好的收率制备出目标炔酰胺. 其缺点是仅限于位阻较 小的内炔酰胺和啞唑烷酮类炔酰胺的制备, 而且二级酰 胺原料必须大大过量, 造成了一定的浪费.

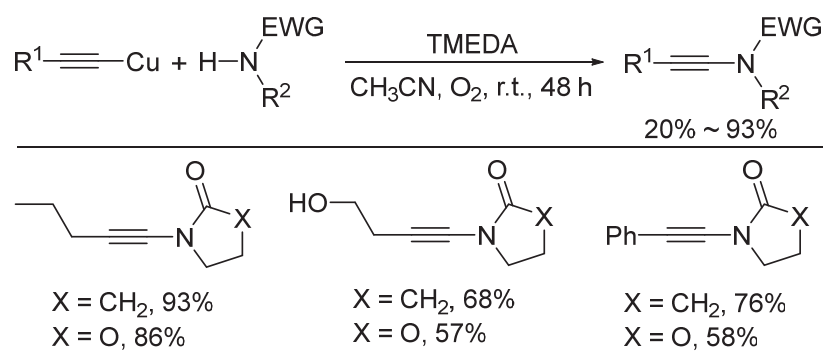

图式 35 炔基铜试剂发生氧化偶联反应合成炔酰胺

Scheme 35 Synthesis of ynamides by oxidative coupling with alkynylcopper reagents

2011 年, Sueda 等 ${ }^{[49]}$ 首次报道了合成 $N$-炔基酰亚胺 的方法(Scheme 36). 反应体系中使用四芳基铋盐作为 炔烃前体, 在铜催化下与酰亚胺合成 $N$-炔基酰亚胺. $N$ 炔基酰亚胺在 TBAF 作用下脱去 TMS, 可以高收率得到 相应端炔酰胺. 但是芳基铋盐较难制备且不易保存，其 合成成本较高.

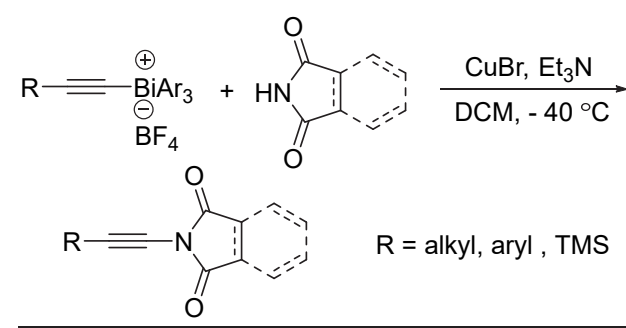

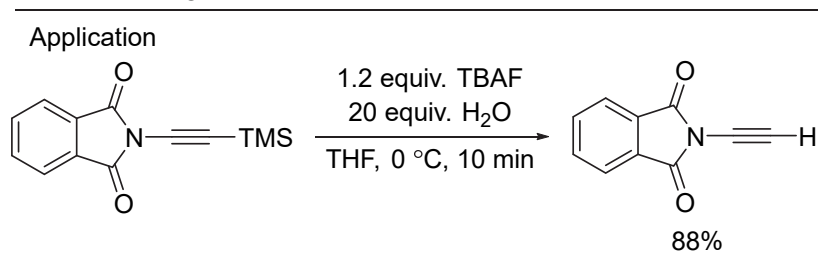

图式 $36 N$-炔基酰亚胺的首次合成 Scheme 36 The first synthesis of $N$-alkynyl imides

\section{$2.4 N$-亲核试剂与端炔合成内炔酰胺}

1985 年, Balsamo 和 Domiano 等 ${ }^{[50]}$ 首次用过渡金属 协助的方法合成炔酰胺(Scheme 37), 反应中需要加入 当量的 CuI.

2008 年以前, 以炔溴和二级酰胺在催化或化学计 量的铜(I)盐存在下合成炔酰胺是最有效的方法. 但是原 料炔溴的制备较为麻烦，且具有较强的催泪性质，也易 爆炸，因此寻找一种更安全有效合成炔酰胺的方法是科 学家亟待解决的问题. 2008 年, Stahl 小组 ${ }^{[51]}$ 发现可以用 
<smiles>CCOC(=O)OCCOC(=O)OC1CC(CI)N1C#CC#C[C-][IH]CC1CC(=O)N1</smiles>

图式 37 过渡金属催化合成炔酰胺

Scheme 37 Synthesis of ynamides by transition metal catalysis

末端炔烃代替炔澳, 与二级酰胺在铜催化下通过氧化偶 联反应合成炔酰胺(Scheme 38). 在氯化铜为催化剂, 碳 酸钠为碱, 甲苯为溶剂, 氧气作为氧化剂的条件下, 端 炔可与二级酰胺通过氧化偶联反应生成内炔酰胺. 该方 法的优点是利用氧气为氧化剂一步合成所需的内炔酰 胺. 其缺点是所用酰胺需要大大过量, 并且炔烃需要溶 于甲苯溶剂中缓慢滴加才能获得良好收率的内炔酰胺,
否则会生成大量的炔烃二聚物. 为克服 Stahl 小组提出 的制备炔酰胺方法中的缺点，Mizuno 等[52]发现末端炔 烃和酰胺可以在氢氧化铜催化下选择性地发生氧化偶 联生成炔酰胺 (Scheme 39). 该方法操作更简单，直接将 廉价易得的氢氧化铜以及两种原料混合摚拌即可获得 各种内炔酰胺. 反应中不需要添加配体, 也无需添加过 量酰胺即可完成反应. 其中, 水为唯一的副产物, 反应 绿色环保.

2012 年, 夏成峰小组 ${ }^{[53]}$ 也报道了一种使用末端炔 烃来合成内炔酰胺的方法, 即在 $\mathrm{KCl}$ 存在下, 氧化铜介 导的末端炔烃与噁唑烷酮直接偶联合成炔酰胺 (Scheme 40). 该方法的缺点是，只适用于噁唑烷酮类化合物，反 应普适性差.

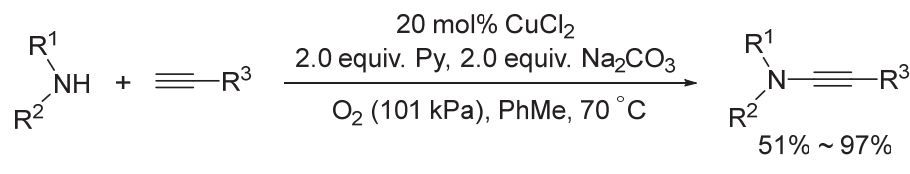
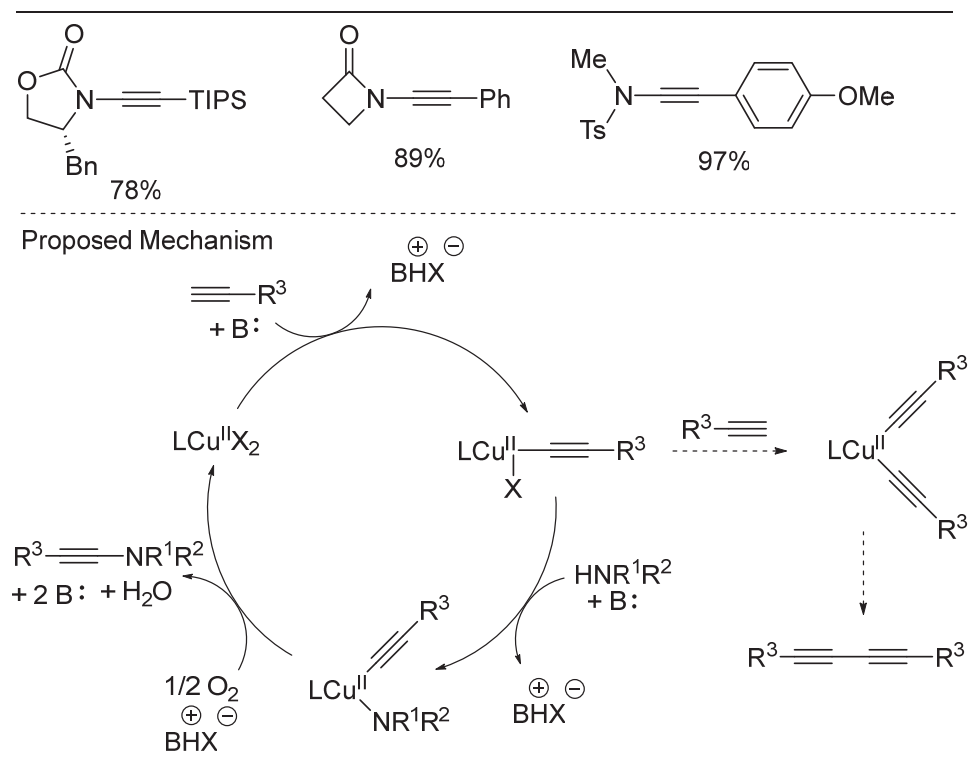

图式 38 炔烃的氧化胺化反应合成炔酰胺

Scheme 38 Synthesis of ynamides by oxidative amidation of alkynes

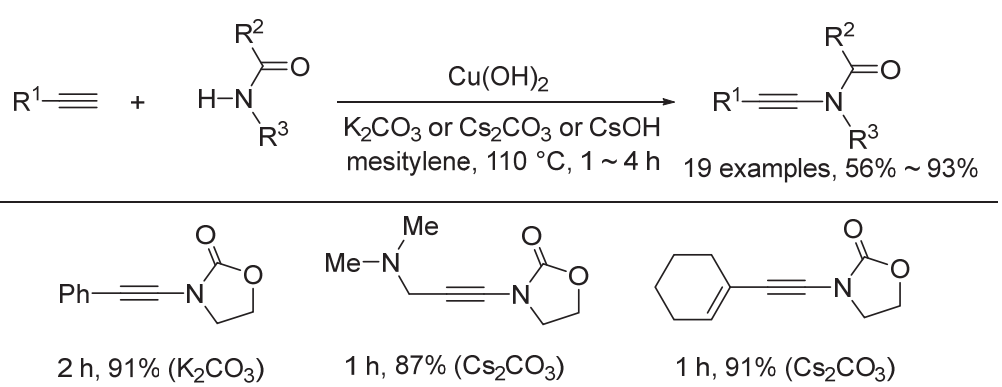
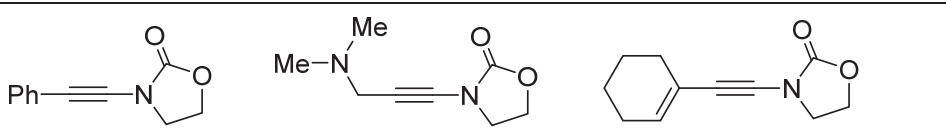

$2 \mathrm{~h}, 91 \%\left(\mathrm{~K}_{2} \mathrm{CO}_{3}\right)$
$1 \mathrm{~h}, 91 \%\left(\mathrm{Cs}_{2} \mathrm{CO}_{3}\right)$

图式 39 氢氧化铜催化氧化偶联反应合成炔酰胺

Scheme 39 Synthesis of ynamides by oxidative cross-coupling with copper hydroxide 
(a)<smiles>[R]C#[C-]</smiles>

$\mathrm{CuO}, \mathrm{KCl}, 4-\mathrm{PPY}$

$R^{2}$

toluene, $80^{\circ} \mathrm{C}, 36 \mathrm{~h}$

(b)

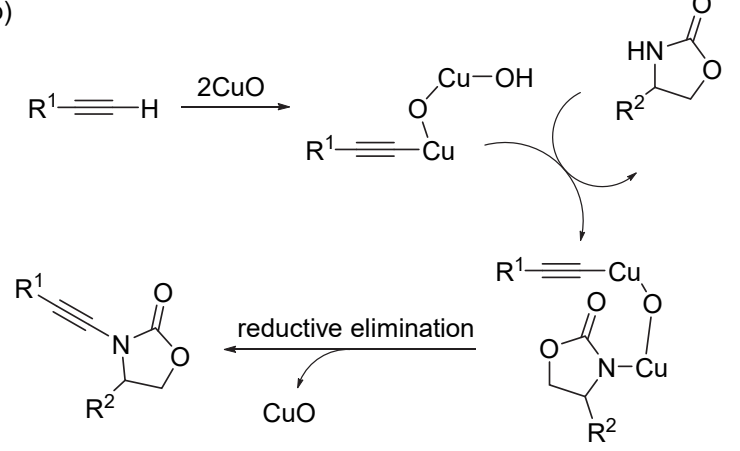

图式 40 (a) 氧化铜介导合成炔酰胺以及(b)可能反应机理

Scheme 40 (a) Synthesis of ynamides by $\mathrm{CuO}$ mediated oxidative coupling and (b) proposed mechanism

Batey 小组 ${ }^{[54]}$ 报道了一种合成酰肼类炔酰胺的方法 (Scheme 41). 在一 $78{ }^{\circ} \mathrm{C}$ 条件下, 炔烃与 ${ }^{n} \mathrm{BuLi}$ 生成炔基 锂试剂, 继而加入偶氮二甲酸二叔丁酯(DBAD), 以中 等收率得到相应的炔酰胺.

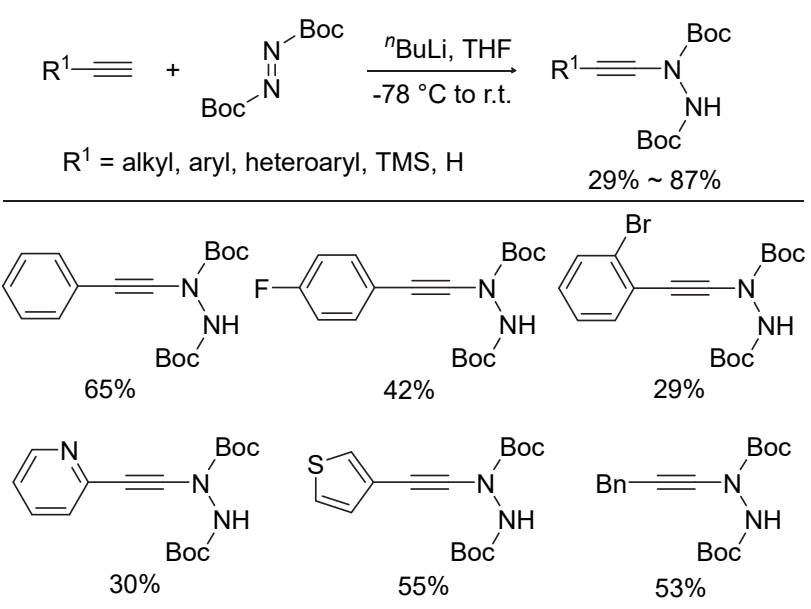

图式41 炔酰肼的合成

Scheme 41 Synthesis of ynehydrazides

2013 年, Bolm 小组 ${ }^{[55]}$ 报道了在氧气条件下, 亚砜亚 胺和炔烃通过氧化偶联反应合成炔酰胺的方法(Scheme 42). 相比 Stahl 小组的工作, 该工作中亚砜亚胺仅仅需 要 2.0 equiv., 且底物适用性更广, 无论是给电子基团还 是吸电子基团都可以容忍, 收率中等偏上.

\section{$3 N$-亲核试剂与卤代烯烃合成炔酰胺}

Evano 课题组一直致力于炔酰胺化学的研

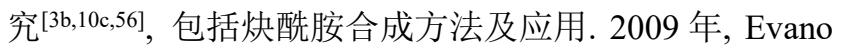
小组 ${ }^{[57]}$ 发现卤代烯烃作为炔烃前体合成炔酰胺更经济 易得. 以 1,1-二溴烯烃作为炔烃前体, 在 60 90 ${ }^{\circ} \mathrm{C}$ 下加
入 4.0 equiv. $\mathrm{Cs}_{2} \mathrm{CO}_{3}$ 和 $18 \mathrm{~mol} \%$ DMEDA，通过 $\mathrm{CuI}$ 的催 化作用，1,1-二溴烯烃和酰胺能以高产率得到炔酰胺 (Scheme 43). 一些可修饰性很高以及 $\mathrm{N}$ 原子上连有大位 阻基团的炔酰胺在该反应体系中都能以很好收率获得, 另外一些复杂结构分子也能兼容.

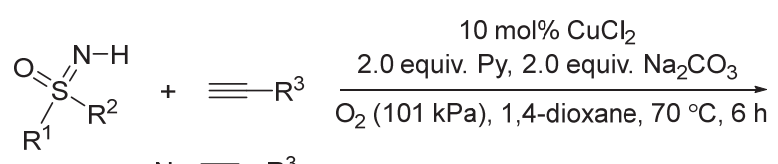

$$
\begin{aligned}
& \mathrm{O}_{\mathrm{O}^{\prime \prime}} \mathrm{N}=\mathrm{R}^{3}
\end{aligned}
$$<smiles>CS(=O)(=NC#Cc1ccc(C#N)cc1)c1ccccc1</smiles><smiles></smiles><smiles>CC[SiH2]C#CN=S(C)(=O)c1ccccc1</smiles>

$84 \%$

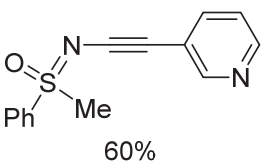

图式 42 氧化偶联反应合成 $N$-炔基亚砜亚胺 Scheme 42 Synthesis of $N$-alkynyl sulfoximines by oxidative coupling of alkynes

通过降低温度，作者分离出中间体溴代烯酰胺，并 将中间体置于等物质的量的 $\mathrm{Cs}_{2} \mathrm{CO}_{3}, \mathrm{DMF}$ 条件下，得到 了相应的炔酰胺. 因此, 作者提出 Scheme 44 所示的反 应机理.

有趣的是 Evano 课题组 ${ }^{[58]}$ 研究了反应条件(如碱、 溶剂及化学计量数等因素)对反应结果的影响. 对这些 条件进行微调, 即可选择性地生成炔酰胺或者 1,1-双烯 酰胺类化合物(Scheme 45).

2011 年, Das 小组报道了在二甲基亚砜(DMSO)中 以 $\left[\mathrm{Cu}(\mathrm{Phen}) \mathrm{PPh}_{3} \mathrm{Br}\right]$ 作为催化剂, 在 $80{ }^{\circ} \mathrm{C}$ 条件下咪唑或 吡唑类化合物与 1,1-二溴烯烃直接合成炔酰胺的方法 (Scheme 46) ${ }^{[59]}$.

2012 年, 梁云小组 ${ }^{[60]}$ 基于 Evano 以 1,1-二溴烯烃作 为炔烃前体合成炔酰胺的方法，报道了一种以 1,2-二溴 烯烃为炔烃前体，在铜催化条件下与磺酰胺直接合成炔 酰胺的方法(Scheme 47).

2014 年，陈正旺小组 ${ }^{[61]}$ 报道了以 2-溴-1-碘烯烃和 氨基甲酸酯为原料，在纳米 $\mathrm{Cu}_{2} \mathrm{O}$ 催化条件下合成炔酰 胺的方法(Scheme 48). 相比炔烃, 原料 2-溴-1-碘烯烃类 化合物的制备更为复杂，限制了该方法的应用。

最近, Anderson 等 ${ }^{[62]}$ 报道了一种合成双氮炔酰胺的 

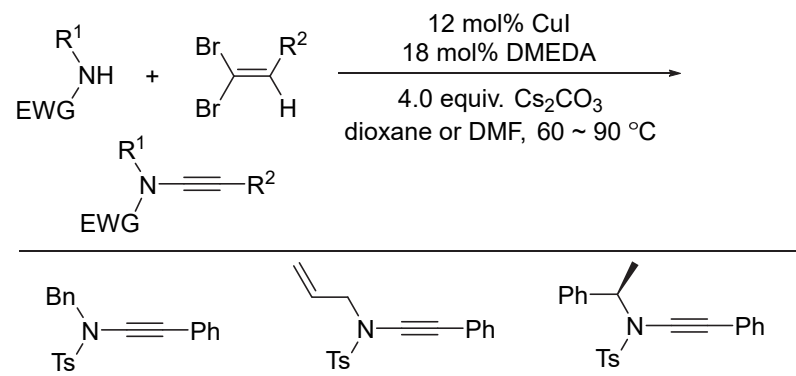

$60{ }^{\circ} \mathrm{C}, 24 \mathrm{~h}, 83 \%$

$60{ }^{\circ} \mathrm{C}, 48 \mathrm{~h}, 81 \%$

$60{ }^{\circ} \mathrm{C}, 24 \mathrm{~h}, 83 \%$
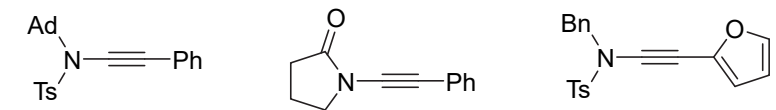

$60{ }^{\circ} \mathrm{C}, 24 \mathrm{~h}, 77 \%$

$60{ }^{\circ} \mathrm{C}, 24 \mathrm{~h}, 80 \%$

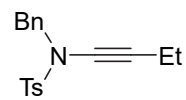

$70{ }^{\circ} \mathrm{C}, 24 \mathrm{~h}, 67 \%$

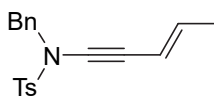

$70{ }^{\circ} \mathrm{C}, 24 \mathrm{~h}, 84 \%$

$60{ }^{\circ} \mathrm{C}, 48 \mathrm{~h}, 88 \%$

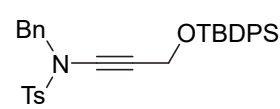

$70{ }^{\circ} \mathrm{C}, 48 \mathrm{~h}, 64 \%$

Application:
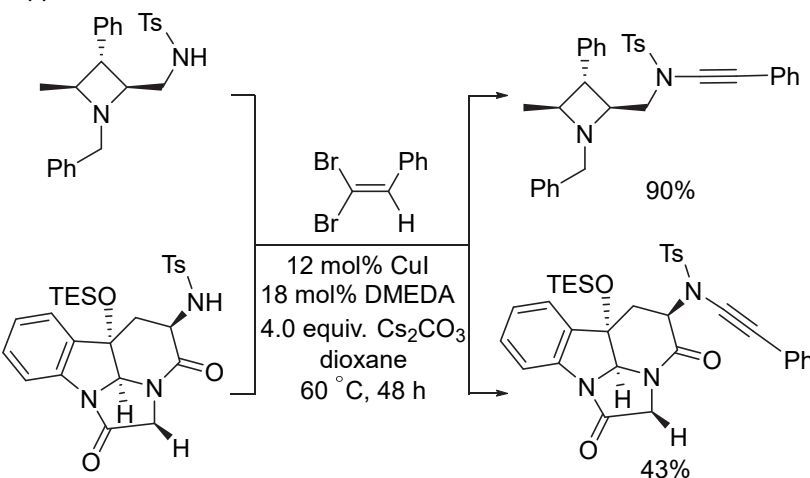

图式 43 以 1,1-二溴烯烃为原料合成炔酰胺

Scheme 43 Synthesis of ynamides with 1,1-dibromo-1-alkenes

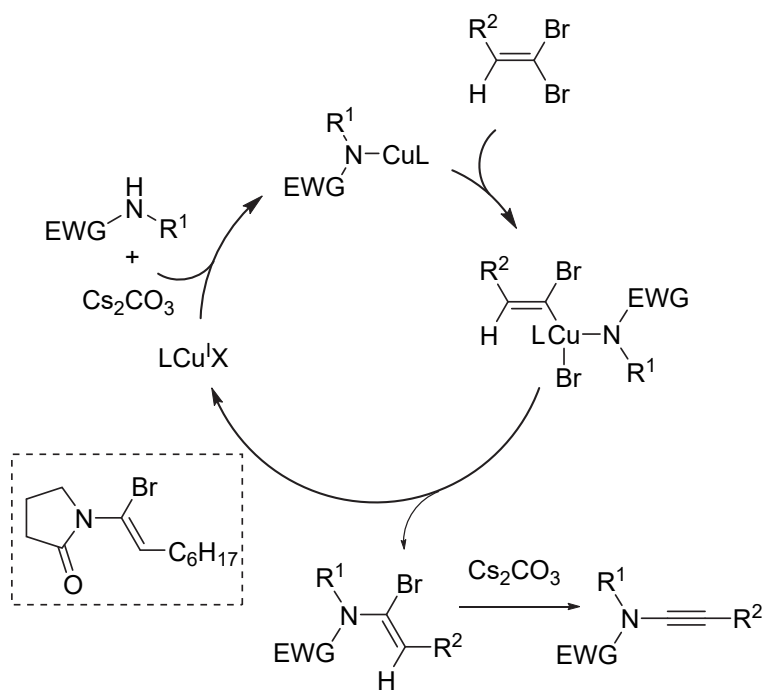

图式 44 可能反应机理

Scheme 44 Proposed mechanism

方法. 1,1-二溴烯酰胺在 $\mathrm{CuI}$ 为催化剂, 1,10-phen 为配体, $\mathrm{Cs}_{2} \mathrm{CO}_{3}$ 为碱, $\mathrm{THF}$ 为溶剂, $60{ }^{\circ} \mathrm{C}$ 条件下反应获得双氮炔 酰胺(Scheme 49). 这些双氮炔酰胺是各种环化反应的

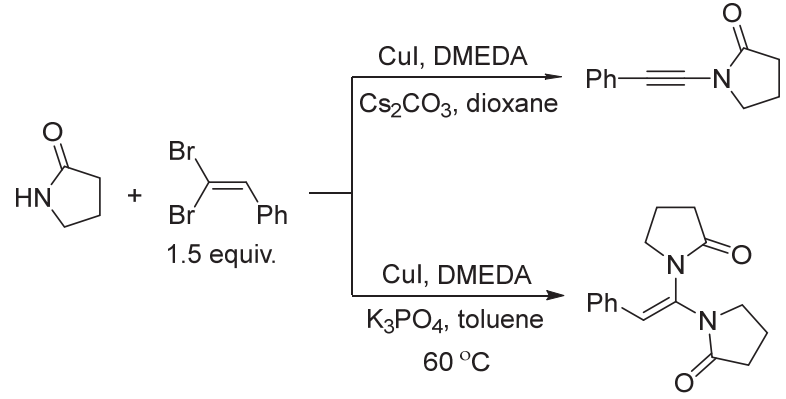

图式 45 选择性生成炔酰胺和双烯酰胺

Scheme 45 Selective synthesis of ynamides and 1,1-dienamides

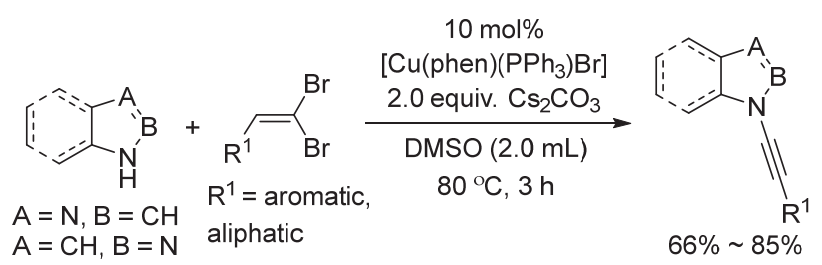

图式 46 咪唑或吡唑类炔酰胺的合成

Scheme 46 Synthesis of ynamides with imidazoles and pyrazoles

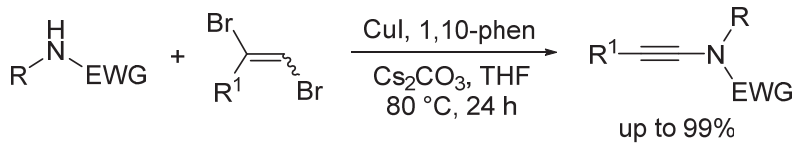

图式 47 以 1,2-二溴烯烃为原料合成炔酰胺

Scheme 47 Synthesis of ynamides with 1,2-dibromo-1-styrenes

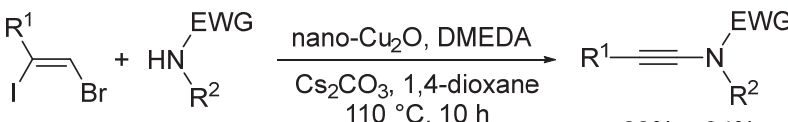

$$
\begin{aligned}
& 69 \% \text { 94\% }
\end{aligned}
$$

图式 48 2-溴-1-碘烯烃为原料合成炔酰胺

Scheme 48 Synthesis of ynamides with 2-bromo-1-iodoalkenes

活性底物. 应用此方法，可以较高收率制备一系列的双 氮炔酰胺.

2016 年, 赵军锋课题组首次发现炔酰胺可以用作 合成酰胺与多肽的缩合试剂 ${ }^{[63]}$, 其最大优点就是不会 诱发 $\alpha$-位手性中心的外消旋化. 后续研究表明炔酰胺缩

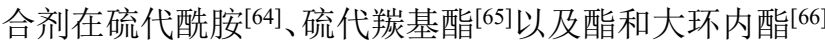
的合成中也表现出了优异的性能，有望成为一种广谱通 用的缩合剂[67]. 因此, 他们希望发展出成本低廉、操作 便捷的炔酰胺合成方法. 经过不断探索，他们创建了从 大宗化工原料一步合成炔酰胺的方法(Scheme 50) ${ }^{[68]}$. 廉价易得的二氯烯烃在碱性条件下与二级磺酰胺(或者 酰胺)直接反应即可得到炔酰胺. 相比具有催泪性质的 二溴烯烃，二氯烯烃作为炔烃前体价格更便宜，更具有 实际应用价值. 并且该方法无需使用过渡金属催化剂和 敏感试剂，操作简单，反应底物普适性好，除了能够制 备一些常见的炔酰胺, 很多结构特殊的炔酰胺以及炔胺 也能够以较好的收率制备. 特别是该方法还适用于端炔 


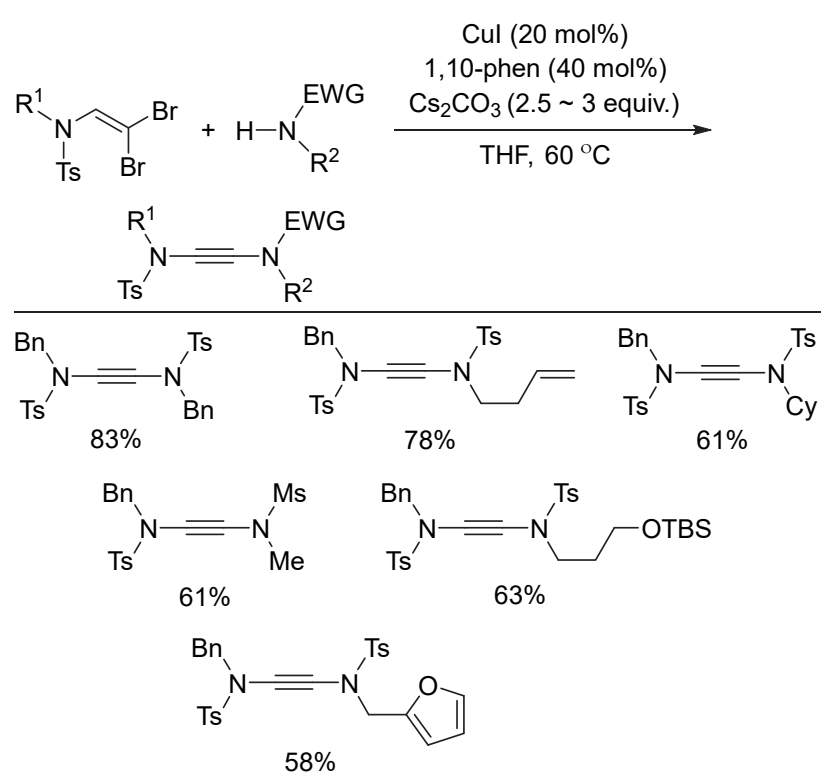

图式 49 双氮炔酰胺的合成

Scheme 49 Synthesis of ynediamides
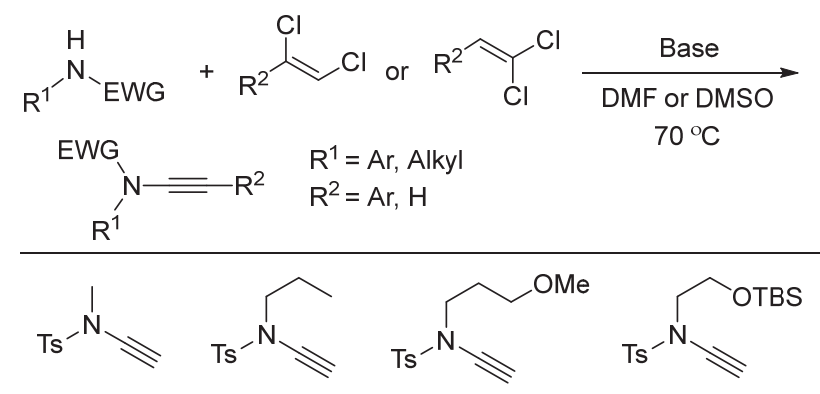

$1 \mathrm{~h}, 98 \% \quad 1 \mathrm{~h}, 95 \% \quad 0.5 \mathrm{~h}, 75 \% \quad 0.5 \mathrm{~h}, 75 \%$<smiles></smiles>

$1 \mathrm{~h}, 83 \%$<smiles>CN(C)C#CPc1ccccc1</smiles>

$1 \mathrm{~h}, 93 \%$<smiles>O=C1OCCN1C#Cc1ccccc1</smiles><smiles>C#Cn1c2ccccc2c2ccccc21</smiles>

$1 \mathrm{~h}, 89 \%$<smiles></smiles>
$26 \mathrm{~h}, 70 \%$

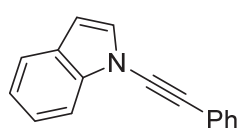

$1 \mathrm{~h}, 88 \%$<smiles>C#CN([13CH3])CCCCCN(C#C)C#C</smiles>

$1 \mathrm{~h}, 68 \%$

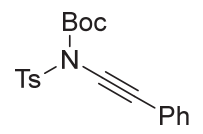

$48 \mathrm{~h}, 55 \%$

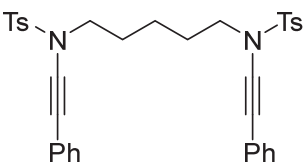

$12 \mathrm{~h}, 88 \%$
图式 50 一步法从 1,1-或者 1,2-二氯烯烃合成炔酰胺

Scheme 50 One step synthesis of ynamides with 1,1- or 1,2dichloroalkenes

酰胺的合成, 且为目前最简单的端炔酰胺合成方法. 该 方法避免了传统端炔酰胺合成方法中先引入保护基再 脱保护的繁琐操作步骤, 原子经济性和步骤经济性高.. 由于 1,1 -二氯乙烯或者 $1,2-$ 二氯乙烯和 $N$-甲基磺酰胺都
是廉价易得的化工原料, 目前在实验室已初步实现公斤 级合成，这为实现端炔酰胺的工业化规模制备奠定了基 础.

\section{4 总结与展望}

炔酰胺由于其特殊的结构和性能受到了有机化学 家的广泛关注，越来越多的炔酰胺参与的新反应逐步被 发掘出来. 发展高效、广谱、简便的炔酰胺合成方法是 炔酰胺能够更好应用于有机合成化学的前提. 尽管化学 家已经开发出了一系列炔酰胺合成方法, 炔烃衍生物与 缺电子胺偶联的方法目前仍是最为简捷直接的策略, 如 铜催化的氧化偶联、高价碘盐法以及卤代烯烃与仲胺偶 联的方法等, 但这些策略也存在一些天生不足, 如底物 拓展范围有限，仅局限于内炔酰胺的合成，反应条件苛 刻或需使用昂贵试剂等. 另外, 大部分端炔酰胺的合成 需要较为繁琐的多步操作也是当前亟待解决的问题. 因 此, 发展便捷经济的炔酰胺合成方法以及实现炔酰胺的 规模化制备仍然是有待化学家继续探索和完善的重要 科学问题.

\section{References}

[1] Zificsak, C. A.; Mulder, J. A.; Hsung, R. P.; Rameshkumar, C.; Wei, L.-L. Tetrahedron 2001, 57, 7575.

[2] Zaugg, H.; Swett, L.; Stone, G. J. Org. Chem. 1958, 23, 1389.

[3] (a) DeKorver, K. A.; Li, H.; Lohse, A. G.; Hayashi, R.; Lu, Z.; Zhang, Y.; Hsung, R. P. Chem. Rev. 2010, 110, 5064.

(b) Evano, G.; Coste, A.; Jouvin, K. Angew. Chem., Int. Ed. 2010, $49,2840$.

[4] (a) Lynch, C. C.; Sripada, A.; Wolf, C. Chem. Soc. Rev. 2020, 49, 8543 .

(b) Luo, J.; Chen, G.-S.; Chen, S.-J.; Yu, J.-S.; Li, Z.-D.; Liu, Y.-L. ACS Catal. 2020, 10, 13978.

(c) Li, X.; Jiang, M.; Zhan, T.; Cao, W.; Feng, X. Chem Asian J. 2020, 15, 1953.

(d) Ito, T.; Harada, S.; Homma, H.; Takenaka, H.; Hirose, S.; Nemoto, T. J. Am. Chem. Soc. 2021, 143, 604.

(e) Li, X.; Zeng, H.; Lin, L.; Feng, X. Org. lett. 2021, 23, 2954.

(f) Zhu, B.-H.; Zhang, Y.-Q.; Xu, H.-J.; Li, L.; Deng, G.-C.; Qian, P.-C.; Deng, C.; Ye, L.-W. ACS Catal. 2021, 11, 1706.

[5] (a) Zhou, B.; Tan, T.-D.; Zhu, X.-Q.; Shang, M.; Ye, L.-W. ACS Catal. 2019, 9, 6393.

(b) Li, X.; Sun, Y.; Zhang, L.; Peng, B. Chin. J. Org. Chem. 2016, 36, 2530 (in Chinese).

(李晓锦, 孙艳, 张否, 彭勃, 有机化学, 2016, 36, 2530.)

(c) Debrauwer, V.; Turlik, A.; Rummler, L.; Prescimone, A.; Blanchard, N.; Houk, K. N.; Bizet, V. J. Am. Chem. Soc. 2020, 142, 11153.

(d) An, D.; Zhang, W.; Pan, B.; Zhao, Y. Eur. J. Org. Chem. 2020, $2021,314$.

(e) Chen, Z.; Nie, X. D.; Sun, J. T.; Yang, A. M.; Wei, B. G. Org. Biomol. Chem. 2021, 19, 2492.

(f) Gao, E.; Peng, C.; Zhang, J.; Wang, X. N.; Chang, J. Org. Biomol. Chem. 2021, 19, 2182.

(g) Nie, X. D.; Han, X. L.; Sun, J. T.; Si, C. M.; Wei, B. G.; Lin, G. Q. J. Org. Chem. 2021, 86, 3433.

(h) Sarathkumar, S.; Kavala, V.; Yao, C. F. Org. lett. 2021, 23, 1960.

[6] (a) Wang, X. N.; Yeom, H. S.; Fang, L. C.; He, S.; Ma, Z. X.; Kedrowski, B. L.; Hsung, R. P. Acc. Chem. Res. 2014, 47, 560. 
(b) Pan, F.; Shu, C.; Ye, L. W. Org. Biomol. Chem. 2016, 14, 9456.

(c) Duret, G.; Le Fouler, V.; Bisseret, P.; Bizet, V.; Blanchard, N. Eur. J. Org. Chem. 2017, 6816.

(d) Zhou, X.; Liang, Z.; Wang, X.-N. Chin. J. Org. Chem. 2021, 41, 1288 (in Chinese).

(周欣悦, 梁宗显, 王晓娜, 有机化学, 2021, 41, 1288.)

(e) Ye, L.-W.; Li, L.; Luo, W.-F. Synlett 2021, 32, 1302.

(f) Chen, Y. B.; Qian, P. C.; Ye, L. W. Chem. Soc. Rev. 2020, 49, 8897.

(g) Liao, Y.; Zhu, L.; Yu, Y.; Chen, G.; Huang, X. Chin. J. Org. Chem. 2017, 37, 2785 (in Chinese).

(廖云, 朱磊, 俞颖华, 陈贵, 黄学良, 有机化学, 2017, 37, 2785.) (h) Zhu, C.; Feng, J.; Zhang, J. Chin. J. Org. Chem. 2017, 37, 1165 (in Chinese).

(朱超泽, 冯见君, 张俊良, 有机化学, 2017, 37, 1165.)

(i) Liu, X.; Liu, X.; Ye, L. Chin. J. Org. Chem. 2021, 41, 1207 (in Chinese).

(刘晓涛, 刘 金金, 叶龙武, 有机化学, 2021, 41, 1207.)

[7] Hu, Y. C.; Zhao, Y.; Wan, B.; Chen, Q. A. Chem. Soc. Rev. 2021, $50,2582$.

[8] (a) Evano, G.; Michelet, B.; Zhang, C. C. R. Chim. 2017, 20, 648.

(b) Li, L.; Tan, T.-D.; Zhang, Y.-Q.; Liu, X.; Ye, L.-W. Org. Biomol. Chem. 2017, 15, 8483 .

(c) Hong, F. L.; Ye, L. W. Acc. Chem. Res. 2020, 53, 2003.

[9] (a) Mahe, C.; Cariou, K. Adv. Synth. Catal. 2020, 362, 4820.

(b) Tan, T. D.; Wang, Z. S.; Qian, P. C.; Ye, L. W. Small Method 2020, 5,2000673

(c) Deng, Y.; Zhang, J.; Bankhead, B.; Markham, J. P.; Zeller, M. Chem. Commun. 2021, 57, 5254.

(d) Zeng, L.; Jin, J.; He, J.; Cui, S. Chem. Commun. 2021, 57, 6792. (e) Zhou, G.; Su, J.; Shang, T.; Wang, X.; Bai, Y.; Yuan, Z.; Zhu, G. Org. Chem. Front. 2021, 8, 4473.

[10] (a) Evano, G.; Jouvin, K.; Coste, A. Synthesis 2012, 45, 17.

(b) Cook, A. M.; Wolf, C. Tetrahedron Lett. 2015, 56, 2377.

(c) Evano, G.; Blanchard, N.; Compain, G.; Coste, A.; Demmer, C. S.; Gati, W.; Guissart, C.; Heimburger, J.; Henry, N.; Jouvin, K.; Karthikeyan, G.; Laouiti, A.; Lecomte, M.; Martin-Mingot, A.; Métayer, B.; Michelet, B.; Nitelet, A.; Theunissen, C.; Thibaudeau, S.; Wang, J.; Zarca, M.; Zhang, C. Chem. Lett. 2016, 45, 574

(d) Wei, L.-L.; Mulder, J. A.; Xiong, H.; Zificsak, C. A.; Douglas, C. J.; Hsung, R. P. Tetrahedron 2001, 57, 459.

(e) Mulder, J. A.; Kurtz, K. C. M.; Hsung, R. P. Synlett 2003, 1379. (f) Zhao, L.; Yang, H.; Li, R.; Tao, Y.; Guo, X. F.; Anderson, E. A.; Whiting, A.; Wu, N. J. Org. Chem. 2021, 86, 1938.

[11] Janousek, Z.; Collard, J.; Viehe, H. G., Angew. Chem., Int. Ed. 1972, 11,917

[12] Joshi, R. V.; Xu, Z.-Q.; Ksebati, M. B.; Kessel, D.; Corbett, T. H.; Drach, J. C.; Zemlicka, J. J. Chem. Soc., Perkin Trans. 1 1994, 1089.

[13] Brückner, D. Tetrahedron 2006, 62, 3809.

[14] Rodríguez, D.; Castedo, L, Saá, C. Synlett 2004, 783.

[15] Rodríguez, D.; Martínez-Esperón, M.; Castedo, L, Saá, C. Synlett 2007, 1963.

[16] Mansfield, S. J.; Campbell, C. D.; Jones, M. W.; Anderson, E. A. Chem. Commun. 2015, 51, 3316

[17] Mansfield, S. J.; Smith, R. C.; Yong, J. R. J.; Garry, O. L.; Anderson, E. A. Org. Lett. 2019, 21, 2918.

[18] Couty, S.; Barbazanges, M.; Meyer, C.; Cossy, J. Synlett 2005, 905

[19] Murch, P.; Williamson, B. L.; Stang, P. J. Synthesis 1994, 1255.

[20] (a) Witulski, B.; Stengel, T. Angew. Chem., Int. Ed. 1998, 37, 489.

(b) Witulski, B.; Gossmann, M. Synlett 2000, 1793

[21] (a) Witulski, B.; Schweikert, T.; Schollmeyer, D.; Nemkovich, N. A. Chem. Commun. 2010, 46, 2953.

(b) Witulski, B.; Lumtscher, J.; Bergsträßer, U. Synlett 2003, 708

(c) Witulski, B.; Stengel, T. Angew. Chem., Int. Ed. 1999, 38, 242.

[22] (a) Rainier, J. D.; Imbriglio, J. E. J. Org. Chem. 2000, 65, 7272.

(b) Rainier, J. D.; Imbriglio, J. E. Org. Lett. 1999, 1, 2037.

[23] Feldman, K. S.; Bruendl, M. M.; Schildknegt, K.; Bohnstedt, A. C.
J. Org. Chem. 1996, 61, 5440.

[24] Witulski, B.; Gößmann, M. Chem. Commun. 1999, 1879.

[25] Souto, J. A.; Becker, P.; Iglesias, A.; Muñiz, K. J. Am. Chem. Soc. 2012, 134, 15505.

[26] Xiang, D.; Li, H.; Zhang, L.; Zhang, Y.; Zhang, Q.; Li, D. Asian J. Org. Chem. 2019, 8, 537 .

[27] Yudasaka, M.; Shimbo, D.; Maruyama, T.; Tada, N.; Itoh, A. Org Lett. 2019, 21, 1098.

[28] Takai, R.; Shimbo, D.; Tada, N.; Itoh, A. J. Org. Chem. 2021, 86, 4699.

[29] Frederick, M. O.; Mulder, J. A.; Tracey, M. R.; Hsung, R. P.; Huang, J.; Kurtz, K. C.; Shen, L.; Douglas, C. J. J. Am. Chem. Soc. 2003, 125,2368

[30] (a) Zhang, Y.; Hsung, R. P.; Tracey, M. R.; Kurtz, K. C.; Vera, E. L. Org. Lett. 2004, 6, 1151

(b) Zhang, X.; Zhang, Y.; Huang, J.; Hsung, R. P.; Kurtz, K. C.; Oppenheimer, J.; Petersen, M. E.; Sagamanova, I. K.; Shen, L.; Tracey, M. R. J. Org. Chem. 2006, 71, 4170.

[31] Hsung., R. P. Org. Synth. 2007, 84, 359.

[32] Zhang, X.; Li, H.; You, L.; Tang, Y.; Hsung, R. P. Adv. Synth. Catal. 2006, 348, 2437.

[33] Dunetz, J. R.; Danheiser, R. L. Org. Lett. 2003, 5, 4011.

[34] Kohnen, A. L. Org. Synth. 2007, 84, 88

[35] Harkat, H.; Borghèse, S.; Nigris, M. D.; Kiselev, S.; Bénéteau, V.; Pale, P. Adv. Synth. Catal. 2014, 356, 3842.

[36] Yao, B.; Liang, Z.; Niu, T.; Zhang, Y. J. Org. Chem. 2009, 74, 4630.

[37] Yang, Y.; Meng, X.; Zhu, B.; Jia, Y.; Cao, X.; Huang, S. Eur. J. Org. Chem. 2019, 1166.

[38] DeKorver, K. A.; Walton, M. C.; North, T. D.; Hsung, R. P. Org. Lett. 2011, 13, 4862 .

[39] Chen, X. Y.; Wang, L.; Frings, M.; Bolm, C. Org. Lett. 2014, 16, 3796.

[40] Anselmi, E.; Le, T. N.; Bouvet, S.; Diter, P.; Pégot, B.; Magnier, E. Eur. J. Org. Chem. 2016, 4423.

[41] (a) Hirano, S.; Tanaka, R.; Urabe, H.; Sato, F. Org. Lett. 2004, 6 , 727.

(b) Hirano, S.; Fukudome, Y.; Tanaka, R.; Sato, F.; Urabe, H. Tetrahedron 2006, 62, 3896

[42] (a) Amanda L. Kohnen, J. R. D., and Rick L.; Danheiser, R. L. Org. Synth. 2007, 84, 88.

(b) Wang, Y. P.; Danheiser, R. L. Tetrahedron Lett. 2011, 52, 2111.

[43] Riddell, N.; Villeneuve, K.; Tam, W. Org. Lett. 2005, 7, 3681.

[44] Betou, M.; Sallustrau, A.; Toupet, L.; Trolez, Y. Tetrahedron Lett. 2015, 56, 4627.

[45] Jia, W.; Jiao, N. Org. Lett. 2010, 12, 2000.

[46] Priebbenow, D. L.; Becker, P.; Bolm, C. Org. Lett. 2013, 15, 6155.

[47] Jouvin, K.; Couty, F.; Evano, G. Org. Lett. 2010, 12, 3272.

[48] Jouvin, K.; Heimburger, J.; Evano, G. Chem. Sci. 2012, 3, 756

[49] Sueda, T.; Oshima, A.; Teno, N. Org. Lett. 2011, 13, 3996.

[50] Balsamo, A.; Macchia, B.; Macchia, F.; Rossello, A.; Domiano, P. Tetrahedron Lett. 1985, 26, 4141.

[51] Hamada, T.; Ye, X.; Stahl, S. S. J. Am. Chem. Soc. 2008, 130, 833.

[52] Jin, X.; Yamaguchi, K.; Mizuno, N. Chem. Commun. 2012, 48 , 4974.

[53] Tong, X.; Ni, G.; Deng, X.; Xia, C. Synlett 2012, 23, 2497.

[54] Beveridge, R. E.; Batey, R. A. Org. Lett. 2012, 14, 540

[55] Wang, L.; Huang, H.; Priebbenow, D. L.; Pan, F. F.; Bolm, C. Angew. Chem., Int. Ed. 2013, 52, 3478.

[56] (a) Evano, G.; Lecomte, M.; Thilmany, P.; Theunissen, C. Synthesis 2017, 49, 3183.

(b) Guissart, C.; Luhmer, M.; Evano, G. Tetrahedron 2018, 74, 6727.

[57] Coste, A.; Karthikeyan, G.; Couty, F.; Evano, G. Angew. Chem., Int. Ed. 2009, 48, 4381.

[58] Coste, A.; Couty, F.; Evano, G. Org. Lett. 2009, 11, 4454.

[59] Das, B.; Salvanna, N.; Reddy, G. C.; Balasubramanyam, P. Tetrahedron Lett. 2011, 52, 6497.

[60] Yang, Y.; Zhang, X.; Liang, Y. Tetrahedron Lett. 2012, 53, 6557. 
[61] Xue, J.; Luo, M.-T.; Wen, Y.-L.; Ye, M.; Liu, L.-X.; Chen, Z.-W. Synthesis 2014, 46, 3191.

[62] Mansfield, S. J.; Christensen, K. E.; Thompson, A. L.; Ma, K.; Jones, M. W.; Mekareeya, A.; Anderson, E. A. Angew. Chem., Int. Ed. 2017, 56, 14428.

[63] Hu, L.; Xu, S.; Zhao, Z.; Yang, Y.; Peng, Z.; Yang, M.; Wang, C.; Zhao, J. J. Am. Chem. Soc. 2016, 138, 13135.

[64] (a) Yang, J.; Wang, C.; Xu, S.; Zhao, J. Angew. Chem., Int. Ed. 2019, 58, 1382.

(b) Yang, J.; Wang, C.; Yao, C.; Chen, C.; Hu, Y.; He, G.; Zhao, J. J. Org. Chem. 2020, 85, 1484.

[65] Yao, C.; Yang, J.; Lu, X.; Zhang, S.; Zhao, J. Org. lett. 2020, 22,
6628.

[66] (a) Yang, M.; Wang, X.; Zhao, J. ACS Catal. 2020, 10, 5230. (b) Wang, X.; Yang, Y.; Zhao, Y.; Wang, S.; Hu, W.; Li, J.; Wang, Z.; Yang, F.; Zhao, J. J. Org. Chem. 2020, 85, 6188.

[67] (a) Xu, S.; Zhao, Z.; Zhao, J. Chin. Chem. Lett. 2018, 29, 1009. (b) Liu, T.; Xu, S.; Zhao, J. Chin. J. Org. Chem. 2021, 41, 873 (in Chinese).

(刘涛, 许泗林, 赵军锋, 有机化学, 2021, 41, 873.)

(a) Tu, Y.; Zeng, X.; Wang, H.; Zhao, J. Org. Lett. 2018, 20, 280.

(b) Zeng, X.; Tu, Y.; Zhang, Z.; You, C.; Wu, J.; Ye, Z.; Zhao, J. J. Org. Chem. 2019, 84, 4458. 